\title{
An analytic approach to infinite-dimensional continuity and Fokker-Planck-Kolmogorov equations
}

\author{
Vladimir I. Bogachev, GiUseppe Da Prato, Michael RöcKNer \\ AND STANISLAV V. SHAPOSHNIKOV
}

\begin{abstract}
We prove a new uniqueness result for solutions to Fokker-PlanckKolmogorov (FPK) equations for probability measures on infinite-dimensional spaces. We consider infinite-dimensional drifts that admit certain finite-dimensional approximations. In contrast to much of the previous work on FPK-equations in infinite dimensions, we include cases with non-constant coefficients in the second order part and also include degenerate cases where these coefficients can even be zero. A new existence result is also proved. Some applications to FPK equations associated with SPDE's are presented.
\end{abstract}

Mathematics Subject Classification (2010): 60H15 (primary); 60J35, 69J60, 47D07 (secondary).

\section{Introduction}

In this paper we study the Cauchy problem for infinite-dimensional Fokker-PlanckKolmogorov equations of the form $\partial_{t} \mu=L^{*} \mu$ for bounded Borel measures $\mu$ on the space $\mathbb{R}^{\infty} \times\left(0, T_{0}\right)$, where $\mathbb{R}^{\infty}$ is the countable power of $\mathbb{R}$ with the product topology, and second order operators

$$
L \varphi=\sum_{i, j} a^{i j} \partial_{x_{i}} \partial_{x_{j}} \varphi+\sum_{i} B^{i} \partial_{x_{i}} \varphi
$$

defined on smooth functions of finitely many variables. Then $A=\left(a^{i j}\right)$ is called the diffusion matrix and $B=\left(B^{i}\right)$ is called the drift coefficient. Such equations arise in many applications and have been intensively studied in the last decades. In particular, they are satisfied by transition probabilities of infinite-dimensional diffusions, which is an important motivation for this paper. The finite-dimensional case has

This work was supported by the RFBR projects 12-01-33009, 14-01-00237, 14-01-91158, 14-0190406-Ukr-f-a, the Simons-IUM fellowship of the Simons Foundation, and by the DFG through the program SFB 701 at the University of Bielefeld. It was completed during a visit to the De Giorgi Center of the Scuola Normale Superiore in Pisa.

Received March 10, 2013; accepted in revised version October 11, 2013. 
been studied in depth by many authors (see the recent surveys [11] and [13]); in particular, there is an extensive literature on regularity and uniqueness of solutions to Fokker-Planck-Kolmogorov equations for measures on finite-dimensional spaces, see $[3,9-11,13,19,29,35]$, and the references there. The infinite-dimensional case is considerably less studied, although there is also a vast literature devoted to this case (see, e.g., [5,7,8,16,25,32], and the references there).

The organization of the paper is as follows. In Section 1 we introduce a general class of Fokker-Planck-Kolmogorov equations in infinite dimensions and prove some preliminary results. In Section 2 we prove uniqueness of probability solutions for these equations under a certain approximative condition (which is a condition on all components of the drift term in a certain uniform way), which considerably generalizes our previous uniqueness results in [7] and [8]. The main difference with the finite-dimensional case is that in the latter the global integrability of the coefficients $a^{i j}$ and $B^{i}$ with respect to the solution ensures its uniqueness, but there is no infinite-dimensional analog of this simple sufficient condition. What we prove is only a partial analog (Example 2.1(ii) formally gives a full analog, but the condition on the norm of the whole drift is very restrictive in infinite dimensions). More precisely, we establish two uniqueness results: Theorem 2.3 (nondegenerate diffusion matrices) and Theorem 2.5 that applies also to degenerate equations, in particular to fully degenerate transport (or continuity) equations including the continuity equation associated to $2 d$-Navier-Stokes equation.

In Section 3 we address the question of existence of solutions to our general FPK-equations and prove Theorem 3.1 which implies existence under quite broad assumptions, in particular, for stochastic Navier-Stokes equations over domains in $\mathbb{R}^{d}$ for all dimensions $d$. In Section 2 and Section 3 we also consider examples that include two other types of SPDEs, namely, stochastic reaction diffusion equations on a bounded domain in $\mathbb{R}^{d}$ (Example 2.10) and Burgers equation (Example 2.11) on the interval $(0,1)$; their mixture is considered in Example 2.12. More precisely, we consider the equation with a constant diagonal $A$ and the drift $B$ of the form $B(u)=D^{2} u+D\left(u^{m}\right)-u^{2 l+1}$, where the last term produces some smoothing effect, which enables us to cover the case of arbitrary $m>2$.

The approach and assumptions in this work differ from those in our earlier paper [5], where probabilistic tools were employed. Here we develop a purely analytic approach without stochastic analysis and (for the first time in infinite dimension) also include the case of nonconstant diffusion matrices. The techniques are also different from the ones in [5,7], and [17], where measures on Hilbert spaces were considered, but the essential difference is not the type of infinite-dimensional spaces, but rather the method of proof which could be called approximative Holmgren method, the idea of which is to multiply the original equation by a solution of a certain equation approximating the adjoint equation (but not the exact adjoint equation as in Holmgren's method) and obtain after integration certain estimates (which replace exact equalities in the classical Holmgren method).

Let us illustrate our approach by the one-dimensional case. Suppose that

$$
L u=a D^{2} u+b D u,
$$


where $a \geq 0$ is a constant. We are going to establish the uniqueness of a probability solution to the equation $\partial_{t} \mu=D_{x}^{2}(a \mu)-D_{x}(b \mu)$. Assume that one can approximate $b$ by a nice sequence of smooth vector fields $b_{k}$. For any $s \in\left(0, T_{0}\right)$ and $\psi \in C_{0}^{\infty}\left(\mathbb{R}^{1}\right)$ we can solve the adjoint Cauchy problem

$$
\partial_{t} f_{k}+a D_{x}^{2} f_{k}+b_{k} D_{x} f_{k}=0,\left.\quad f_{k}\right|_{t=s}=\psi .
$$

By the maximum principle $\left|f_{k}\right| \leq \max |\psi|$. Let $\mu=\mu^{1}-\mu^{2}$ be the difference of two different probability solutions $\mu^{1}$ and $\mu^{2}$. Multiplying the equation $\partial_{t} \mu=$ $D_{x}^{2}(a \mu)-D_{x}(b \mu)$ by the function $f_{k}$ and integrating by parts we obtain the equality

$$
\int \psi d \mu_{s}=\int_{0}^{s} \int\left(b-b_{k}\right) D_{x} f_{k} d \mu_{t} d t .
$$

The main difficulty is to prove that the right-hand side tends to zero. We need some estimates on $\left|D_{x} f_{k}\right|$. There are two different cases.

(I) Let $a \neq 0$. Then multiplying the equation for $\mu^{1}+\mu^{2}$ by the function $f_{k}^{2}$, integrating by parts and using the Cauchy inequality we have

$$
\int_{0}^{s} \int a\left|D_{x} f_{k}\right|^{2} d\left(\mu_{t}^{1}+\mu_{t}^{2}\right) d t \leq \max |\psi|^{2}\left(1+a^{-1} \int_{0}^{s} \int\left|b-b_{k}\right|^{2} d\left(\mu_{t}^{1}+\mu_{t}^{2}\right) d t\right) .
$$

Assume that

$$
\lim _{k \rightarrow \infty} \int_{0}^{s} \int\left|b-b_{k}\right|^{2} d\left(\mu_{t}^{1}+\mu_{t}^{2}\right)=0 .
$$

According to the above estimate

$$
\begin{aligned}
\int \psi d \mu_{s} & \leq\left(\int_{0}^{s} \int\left|b-b_{k}\right|^{2} d\left(\mu_{t}^{1}+\mu_{t}^{2}\right) d t\right)^{1 / 2}\left(\int_{0}^{s} \int\left|D_{x} f_{k}\right|^{2} d\left(\mu_{t}^{1}+\mu_{t}^{2}\right) d t\right)^{1 / 2} \\
& \longrightarrow 0 \text { if } k \rightarrow \infty .
\end{aligned}
$$

So we have

$$
\int \psi d \mu_{s} \leq 0
$$

for every $\psi \in C_{0}^{\infty}\left(\mathbb{R}^{1}\right)$, which gives the equality $\mu_{s}=\mu_{s}^{1}-\mu_{s}^{2}=0$.

(II) Let $a=0$. The required estimate can be obtained by the maximum principle. Let us differentiate the equation $\partial_{t} f_{k}+b_{k} D_{x} f_{k}=0$ in $x$ and multiply the result by the function $D_{x} f_{k}$. We obtain

$$
\partial_{t} v_{k}+b_{k} D_{x} v_{k}+2 D_{x} b_{k} v=0, \quad v_{k}=\left|D_{x} f_{k}\right|^{2} / 2 .
$$

The main difficulty is that in general the functions $D_{x} b_{k}$ are not bounded from above uniformly in $k$. Assume that there exists a sequence of smooth functions $V_{k} \geq 1$ on $\mathbb{R}^{1}$ such that

$$
L V_{k}=b_{k} D_{x} V_{k} \leq\left(C-2 D_{x} b_{k}\right) V_{k}
$$


for some number $C$ and every $k$. Then the function $w_{k}=v_{k} / V_{k}$ satisfies the inequality

$$
\partial_{t} w_{k}+b_{k} D_{x} w_{k}+C w_{k} \geq 0
$$

and the maximum principle gives the estimate $w_{k}(x, t) \leq e^{C(s-t)} \max w_{k}(x, 0)$. So we have

$$
\left|D_{x} f_{k}(x, t)\right| \leq e^{C(s-t) / 2} \sqrt{V_{k}(x)} \max \left|D_{x} \psi\right| .
$$

Assume that

$$
\lim _{k \rightarrow \infty} \int_{0}^{T_{0}} \int\left|b-b_{k}\right| \sqrt{V_{k}} d\left(\mu_{t}^{1}+\mu_{t}^{2}\right) d t=0
$$

Then, letting $k \rightarrow \infty$, we arrive at the inequality $\int \psi d \mu_{s} \leq 0$. Hence $\mu_{s}=0$.

The same method works equally well also in the infinite-dimensional case, as we shall see below; a more detailed study of the finite-dimensional case is presented in our paper [6].

\section{Framework and preliminaries}

Let us describe our framework. Let $B=\left(B^{i}\right)$ be a sequence of Borel functions on $\mathbb{R}^{\infty} \times\left(0, T_{0}\right)$, where $T_{0}>0$ is fixed, and let $a^{i j}$ be Borel functions on $\mathbb{R}^{\infty} \times\left(0, T_{0}\right)$. Let $A=\left(a^{i j}\right), B=\left(B^{i}\right)$. Let us consider the Cauchy problem

$$
\left\{\begin{array}{l}
\partial_{t} \mu=L^{*} \mu \\
\left.\mu\right|_{t=0}=v
\end{array}\right.
$$

where $L^{*}$ is the formal adjoint operator for a differential operator $L$ defined by

$$
L \varphi(x, t)=\sum_{i, j=1}^{\infty} a^{i j}(x, t) \partial_{x_{i}} \partial_{x_{j}} \varphi(x, t)+\sum_{i=1}^{\infty} B^{i}(x, t) \partial_{x_{i}} \varphi(x, t)
$$

for every smooth function $\varphi$ depending on finitely many coordinates of $x, \partial_{x_{i}} \varphi$ denotes the partial derivative with respect to $x_{i}$. Equations of this form are usually called Fokker-Planck-Kolmogorov equations.

Throughout this paper "a measure" means a bounded signed measure (not necessarily nonnegative, although our principal results will be concerned with probability measures). The total variation of a measure $\mu$ is denoted by $|\mu|$. Let $J$ be an interval in $[0,+\infty)$. We use the standard notation $C\left(\mathbb{R}^{k} \times J\right)$ and $C^{2,1}\left(\mathbb{R}^{k} \times J\right)$ for the class of real continuous functions on $\mathbb{R}^{k} \times J$ and its subclass consisting of all functions $f$ having continuous partial derivatives $\partial_{t} f, \partial_{x_{i}} f$ and $\partial_{x_{i}} \partial_{x_{j}} f$ (the latter will be also denoted by $\left.\partial_{x_{i} x_{j}} f\right)$. Let $C_{b}\left(\mathbb{R}^{k} \times J\right)$ and $C_{b}^{2,1}\left(\mathbb{R}^{k} \times J\right)$ denote the subclasses in these classes consisting of bounded functions and functions $f$ with bounded derivatives $\partial_{t} f, \partial_{x_{i}} f$ and $\partial_{x_{i} x_{j}} f$, respectively, and $C_{0}^{2,1}\left(\mathbb{R}^{k} \times J\right)$ is the 
subspace in $C_{b}^{2,1}\left(\mathbb{R}^{k} \times J\right)$ consisting of functions with compact support in $\mathbb{R}^{k} \times J$. For functions on $\mathbb{R}^{k}$ we use the standard symbols $C_{0}^{2}\left(\mathbb{R}^{k}\right), C_{b}^{2}\left(\mathbb{R}^{k}\right)$ and $C_{0}^{\infty}\left(\mathbb{R}^{k}\right)$ for the classes of twice continuously differentiable functions with compact support, functions with bounded continuous derivatives up to the second order, and infinitely differentiable functions with compact support, respectively.

The inner product in $\mathbb{R}^{n}$ will be denoted by $\left.\langle\cdot, \cdot\rangle\right\rangle$; in the case of $L^{2}$-spaces we write $\langle\cdot, \cdot\rangle_{2}$ for its inner product and the corresponding norm is denoted by $\|\cdot\|_{2}$. The $L^{p}$-norm will be denoted by $\|\cdot\|_{p}$. The norm $\|\cdot\|_{p, k}$ in the Sobolev space $H^{p, k}(U)$ of all functions on a domain $U$ belonging to $L^{p}(U)$ along with their generalized partial derivatives up to order $k$ is defined as the sum of the $L^{p}$-norms of all partial derivatives up to order $k$ (including $k=0$ ).

Let $P_{N}: \mathbb{R}^{\infty} \rightarrow \mathbb{R}^{N}, P_{N} x=\left(x_{1}, \ldots, x_{N}\right)$. Given a function $\varphi$ on $\mathbb{R}^{k}$ we denote by the same symbol the function on $\mathbb{R}^{\infty}$ defined by $\varphi(x):=\varphi\left(P_{k} x\right)$.

We shall consider Borel measures on $\mathbb{R}^{\infty} \times\left(0, T_{0}\right)$ of the form

$$
\mu=\mu_{t}(d x) d t,
$$

where each $\mu_{t}$ is a bounded Borel measure on $\mathbb{R}^{\infty}$ (possibly signed), which means that for every Borel set $B$ in $\mathbb{R}^{\infty}$ the function $t \mapsto \mu_{t}(B)$ is measurable, the function $t \mapsto\left\|\mu_{t}\right\|$ is integrable on $\left(0, T_{0}\right)$ and

$$
\int_{\mathbb{R}^{\infty} \times\left(0, T_{0}\right)} f d \mu=\int_{0}^{T_{0}} \int_{\mathbb{R}^{\infty}} f(x, t) \mu_{t}(d x) d t
$$

for every bounded Borel function $f$ on $\mathbb{R}^{\infty} \times\left(0, T_{0}\right)$; under the stated conditions the latter integral exists.

We shall say that a bounded Borel measure $\mu=\mu_{t}(d x) d t$ on $\mathbb{R}^{\infty} \times\left(0, T_{0}\right)$, where $\left(\mu_{t}\right)_{0<t<T_{0}}$ is a family of bounded Borel measures on $\mathbb{R}^{\infty}$, satisfies the equation

$$
\partial_{t} \mu=L^{*} \mu
$$

if the functions $a^{i j}, B^{i}$ are integrable with respect to the variation $|\mu|$ of $\mu$ and for every $k \geq 1$ and every function $\varphi \in C_{0}^{2,1}\left(\mathbb{R}^{k} \times\left(0, T_{0}\right)\right)$ we have

$$
\int_{0}^{T_{0}} \int_{\mathbb{R}^{\infty}}\left[\partial_{t} \varphi+\sum_{i, j=1}^{\infty} a^{i j} \partial_{x_{i}} \partial_{x_{j}} \varphi+\sum_{i=1}^{\infty} B^{i} \partial_{x_{i}} \varphi\right] d \mu_{t} d t=0 .
$$

It is obvious that it is enough to have this identity for all $\varphi \in C_{0}^{\infty}\left(\mathbb{R}^{k} \times\left(0, T_{0}\right)\right)$.

It will be convenient to assume in some results below that the functions $a^{i j}$ and $B^{i}$ are defined on $\mathbb{R}^{\infty} \times\left[0, T_{0}\right]$. Due to the special form of $\mu$ described above the values of these functions at $t=0$ and $t=T_{0}$ are not important for the equation.

Let $v$ be a bounded Borel measure on $\mathbb{R}^{\infty}$. We say that the measure $\mu$ satisfies the initial condition $\left.\mu\right|_{t=0}=v$ if for every $k \geq 1$ and $\zeta \in C_{0}^{2}\left(\mathbb{R}^{k}\right)$ we have

$$
\lim _{t \rightarrow 0} \int_{\mathbb{R}^{\infty}} \zeta(x) \mu_{t}(d x)=\int_{\mathbb{R}^{d}} \zeta(x) v(d x) .
$$


Clearly, if $\sup _{t}\left\|\mu_{t}\right\|<\infty$, it suffices to have this equality for all $\zeta \in C_{0}^{\infty}\left(\mathbb{R}^{k}\right)$.

We need the following auxiliary lemma.

Lemma 1.1. Let $\mu=\mu_{t}(d x) d t$ be a solution to (1.1) such that $\sup _{t \in\left(0, T_{0}\right)}\left\|\mu_{t}\right\|<$ $\infty$. Assume that $B^{k} \in L^{1}(|\mu|)$ for every $k \in \mathbb{N}$ and let $0<T<T_{0}$. Then for every number $k \geq 1$ and every function $\varphi \in C_{b}\left(\mathbb{R}^{k} \times[0, T]\right) \bigcap C_{b}^{2,1}\left(\mathbb{R}^{k} \times(0, T)\right)$ the equality

$$
\int_{\mathbb{R}^{\infty}} \varphi(x, t) \mu_{t}(d x)=\int_{\mathbb{R}^{\infty}} \varphi(x, 0) \nu(d x)+\int_{0}^{t} \int_{\mathbb{R}^{\infty}}\left[\partial_{s} \varphi+L \varphi\right] d \mu_{s} d s
$$

holds for almost every $t \in[0, T]$. Conversely, (1.2) implies (1.1).

Proof. It is enough to prove this equality in the case where $\varphi(z, t)=0$ if $|z|>R>$ 0 for almost every $t \in[0, T]$. Let $\eta \in C_{0}^{\infty}((0, T))$. According to our definition we have

$$
\int_{0}^{T} \int_{\mathbb{R}^{\infty}}\left[\partial_{t}(\varphi \eta)+L(\varphi \eta)\right] d \mu_{t} d t=0
$$

Thus, we obtain

$$
-\int_{0}^{T} \eta^{\prime}(t) \int_{\mathbb{R}^{\infty}} \varphi(x, t) \mu_{t}(d x) d t=\int_{0}^{T} \eta(t) \int_{\mathbb{R}^{\infty}}\left[\partial_{t} \varphi+L \varphi\right] d \mu_{t} d t .
$$

Hence the function

$$
t \mapsto \int_{\mathbb{R}^{\infty}} \varphi(x, t) \mu_{t}(d x)
$$

on $(0, T)$ has an absolutely continuous version for which

$$
\frac{d}{d t} \int_{\mathbb{R}^{\infty}} \varphi(x, t) \mu_{t}(d x)=\int_{\mathbb{R}^{\infty}}\left[\partial_{t} \varphi+L \varphi\right] d \mu_{t} .
$$

Therefore, for some constant $C \in \mathbb{R}$ the equality

$$
\int_{\mathbb{R}^{\infty}} \varphi(x, t) \mu_{t}(d x)=C+\int_{0}^{t} \int_{\mathbb{R}^{\infty}}\left[\partial_{s} \varphi+L \varphi\right] d \mu_{s} d s
$$

holds for almost every $t \in[0, T]$. Note that $\varphi(x, t)$ converges uniformly to $\varphi(x, 0)$ as $t \rightarrow 0$. Moreover, we have

$$
\lim _{t \rightarrow 0} \int_{\mathbb{R}^{\infty}} \varphi(x, 0) \mu_{t}(d x)=\int_{\mathbb{R}^{\infty}} \varphi(x, 0) v(d x) .
$$

It follows that

$$
C=\int_{\mathbb{R}^{\infty}} \varphi(x, 0) v(d x),
$$

which completes the proof of one implication. The converse is, however, obvious. 
Remark 1.2. Let $k \in \mathbb{N}$. If $\varphi(\cdot, t)=\psi \in C_{b}^{2}\left(\mathbb{R}^{k}\right)$ for every $t \in[0, T], T<T_{0}$, then by (1.2) we have

$$
\int_{\mathbb{R}^{\infty}} \psi(x) \mu_{t}(d x)=\int_{\mathbb{R}^{\infty}} \psi(x) v(d x)+\int_{0}^{t} \int_{\mathbb{R}^{\infty}} L \psi(x, s) \mu_{s}(d x) d s
$$

for almost all $t \in[0, T]$. Moreover, if $J_{\psi}^{\mu}$ denotes the set of all $t \in[0, T]$ such that equality (1.3) holds, then the closure of $J_{\psi}^{\mu}$ coincides with $[0, T]$ and the restriction of the mapping

$$
t \mapsto \int_{\mathbb{R}^{\infty}} \psi(x) \mu_{t}(d x)
$$

to $J_{\psi}^{\mu}$ is continuous, since the right-hand side of (1.3) is continuous in $t$.

Remark 1.3. Let $\varphi$ be as in Lemma 1.1. and assume that $T \in J_{\varphi(\cdot, T)}^{\mu}$. Then equality (1.2) holds with $t=T$. Indeed, $\varphi(x, t)$ converges uniformly to $\varphi(x, T)$ as $t \rightarrow T$. Let $I$ be the set of all $t \in[0, T]$ such that equality (1.2) holds. Let us take a sequence $t_{n} \in J_{\varphi(\cdot, T)}^{\mu} \bigcap I$ such that $\lim _{n \rightarrow \infty} t_{n}=T$. Then we have

$$
\lim _{n \rightarrow \infty} \int_{\mathbb{R}^{\infty}} \varphi\left(x, t_{n}\right) \mu_{t_{n}}(d x)=\int_{\mathbb{R}^{\infty}} \varphi(x, T) \mu_{T}(d x)
$$

and equality (1.2) holds for each $t_{n}$. Letting $n \rightarrow \infty$, we obtain equality (1.2) with $t=T$.

\section{Uniqueness of probability solutions}

In this section we establish two different uniqueness results: first we consider nondegenerate diffusion matrices and then turn to the general case that includes fully degenerate equations. We start with stating our assumptions about $A$ and $B$.

(A) $a^{i j}=a^{j i}$, each function $a^{i j}$ depends only on the variables $t, x_{1}, x_{2}, \ldots, x_{\max \{i, j\}}$ and is continuous and for every natural number $N$ the matrix $A_{N}=\left(a^{i j}\right)_{1 \leq i, j \leq N}$ satisfies the following condition:

- there exist positive numbers $\gamma_{N}, \lambda_{N}$ and $\beta_{N} \in(0,1]$ such that for all $x, y \in \mathbb{R}^{N}$ and $t \in\left[0, T_{0}\right]$ one has

$$
\gamma_{N}|y|^{2} \leq\left\langle A_{N}(x, t) y, y\right\rangle \leq \gamma_{N}^{-1}|y|^{2}, \quad\left\|A_{N}(x, t)-A_{N}(y, t)\right\| \leq \lambda_{N}|x-y|^{\beta_{N}},
$$

where $\|\cdot\|$ is the operator norm and $|\cdot|$ is the standard Euclidean norm.

Let $v$ be a Borel probability measure on $\mathbb{R}^{\infty}$ and let $\mathcal{P}_{v}$ be some convex set of probability solutions $\mu=\mu_{t}(d x) d t$ to $(1.1)$, i.e., $\mu_{t} \geq 0$ and $\mu_{t}\left(\mathbb{R}^{\infty}\right)=1$ for every $t \in\left(0, T_{0}\right)$, such that $\left|B^{k}\right| \in L^{2}(\mu)$ for each $\bar{k} \in \mathbb{N}$ and the following condition holds: 
(B) for every $\varepsilon>0$ and every natural number $d$ there exist a natural number $N=$ $N(\varepsilon, d) \geq d$ and a $C_{b}^{2,1}$-mapping $\left(b_{\varepsilon}^{k}\right)_{k=1}^{N}: \mathbb{R}^{N} \times\left[0, T_{0}\right] \rightarrow \mathbb{R}^{N}$ such that

$$
\int_{0}^{T_{0}} \int_{\mathbb{R}^{\infty}}\left|A_{N}(x, t)^{-1 / 2}\left(B_{N}(x, t)-b_{\varepsilon}\left(x_{1}, \ldots, x_{N}, t\right)\right)\right|^{2} \mu_{t}(d x) d t<\varepsilon,
$$

where $B_{N}=\left(B^{1}, \ldots, B^{N}\right)$. We do not indicate dependence on $d$ where it is meant.

Let us illustrate condition (B) by several examples.

We shall use the following notation: given a sequence $\kappa=\left(\kappa_{n}\right)_{n \geq 1}$ of positive numbers, the weighted Hilbert space

$$
l_{\kappa}^{2}=\left\{x=\left(x_{n}\right):\|x\|_{l_{\kappa}^{2}}^{2}=\sum_{n=1}^{\infty} \kappa_{n} x_{n}^{2}<\infty\right\}
$$

will be equipped with the inner product $\langle x, y\rangle_{\kappa}=\sum_{n=1}^{\infty} \kappa_{n} x_{n} y_{n}$.

\section{Example 2.1.}

(i) Let $B^{k}$ depend only on the variables $t, x_{1}, x_{2}, \ldots, x_{k}$. Then in order to ensure our condition (B) we need only the inclusion $\left|B^{k}\right| \in L^{2}(\mu)$ for all $k \geq 1$. Indeed, we set $N=d$ and approximate each function $B^{k}$ separately.

(ii) Let $\alpha=\left(\alpha_{k}\right)_{k \geq 1}, \alpha_{k}>0$ for each $k \in \mathbb{N}$ and $1 / \alpha:=\left(\alpha_{k}^{-1}\right)_{k \geq 1}$. Suppose that $a^{i j}$ satisfy condition (A) and there exists a positive number $C$ independent of $N$ such that

$$
\left|A_{N}(x, t)^{-1 / 2} y\right| \leq C\|y\|_{l_{1 / \alpha}^{2}}
$$

for all $x, t$ and $y=\left(y_{1}, y_{2}, \ldots, y_{N}, 0,0, \ldots\right)$. For example, this is true if $a^{i j}=0$ for $i \neq j$ and $a^{i i}=\alpha_{i}$.

Let $\left(B^{k}(x, t)\right) \in l_{1 / \alpha}^{2}$ for $\mu$-almost every $(x, t)$ and let $\|B\|_{l_{1 / \alpha}^{2}} \in L^{2}(\mu)$. For every $\varepsilon>0$ and every natural number $d$ we pick a number $M>d$ such that

$$
\sum_{k=M+1}^{\infty} \int_{0}^{T_{0}} \int_{\mathbb{R}^{\infty}} \alpha_{k}^{-1}\left|B^{k}\right|^{2} d \mu_{t} d t<\varepsilon / 2 .
$$

Then for every $B^{k}$ we find a smooth function $b_{\varepsilon}^{k}$ depending on the first $n_{k}$ variables such that

$$
\int_{0}^{T_{0}} \int_{\mathbb{R}^{\infty}} \alpha_{k}^{-1}\left|B^{k}-b_{\varepsilon}^{k}\right|^{2} d \mu_{t} d t<\varepsilon(2 M)^{-1}, \quad k=1, \ldots, M .
$$

Set $N=\max \left\{M, n_{1}, n_{2}, \ldots, n_{M}\right\}$ and $b_{\varepsilon}^{k} \equiv 0$ for $k>N$. Then

$$
\begin{aligned}
& \sum_{k=1}^{N} \int_{0}^{T_{0}} \int_{\mathbb{R}^{\infty}} \alpha_{k}^{-1}\left|B^{k}-b_{\varepsilon}^{k}\right|^{2} d \mu_{t} d t \\
& =\sum_{k=1}^{M} \int_{0}^{T_{0}} \int_{\mathbb{R}^{\infty}} \alpha_{k}^{-1}\left|B^{k}-b_{\varepsilon}^{k}\right|^{2} d \mu_{t} d t+\sum_{k=M+1}^{N} \int_{0}^{T_{0}} \int_{\mathbb{R}^{\infty}} \alpha_{k}^{-1}\left|B^{k}\right|^{2} d \mu_{t} d t<\varepsilon .
\end{aligned}
$$


(iii) Finally, for $a^{i j}$ as in (ii), we can combine both examples. Let $B=G+F$, where $G^{k}, F^{k} \in L^{2}(\mu), G^{k}(x, t)=G^{k}\left(x_{1}, x_{2}, \ldots, x_{k}, t\right), F(x, t) \in l_{1 / \alpha}^{2}$ and $\|F\|_{1 / \alpha} \in L^{2}(\mu)$. Obviously, for given $B^{k}$ of this type the set of all probability solutions $\mu=\mu_{t}(d x) d t$ to (1.1) satisfying the previous integrability conditions is convex.

\section{Remark 2.2.}

(i) Condition (B) is equivalent to the following condition: there exist an increasing sequence $N_{l} \rightarrow+\infty$ and $C_{b}^{2,1}$-mappings $b_{l}: \mathbb{R}^{N_{l}} \times\left[0, T_{0}\right] \rightarrow \mathbb{R}^{N_{l}}$ such that

$$
\lim _{l \rightarrow \infty} \int_{0}^{T_{0}} \int_{\mathbb{R}^{\infty}}\left|A_{N_{l}}(x, t)^{-1 / 2}\left(B_{N_{l}}(x, t)-b_{l}\left(x_{1}, \ldots, x_{N_{l}}, t\right)\right)\right|^{2} \mu_{t}(d x) d t=0 .
$$

(ii) Assume that $a^{i j}=\delta^{i j}$. Let $\widetilde{P}_{N}(x, t)=\left(P_{N} x, t\right)$ and let $\mathbb{E}_{\mu}\left[\cdot \mid \widetilde{P}_{N}=(x, t)\right]$ be the corresponding conditional expectation. Then condition (B) is equivalent to the following condition: for every $\varepsilon>0$ and every natural number $d$ there exists a natural number $N \geq d$ such that

$$
\int_{0}^{T_{0}} \int_{\mathbb{R}^{\infty}} \sum_{k=1}^{N}\left|B^{k}(x, t)-\mathbb{E}_{\mu}\left[B^{k} \mid \widetilde{P}_{N}=(x, t)\right]\right|^{2} \mu_{t}(d x) d t<\varepsilon .
$$

This condition is known in Euclidean quantum field theory as the HøeghKrohn condition (see [1]) and has been used, e.g., to prove Markov uniqueness for semigroups (see [33]).

Theorem 2.3. Assume that conditions (A) and (B) hold. Then the set $\mathcal{P}_{v}$ contains at most one element.

Proof. Assume that two measures $\sigma^{1}=\sigma_{t}^{1} d t$ and $\sigma^{2}=\sigma_{t}^{2} d t$ belong to $\mathcal{P}_{v}$. By our assumption about $\mathcal{P}_{\nu}, \sigma=\left(\sigma^{1}+\sigma^{2}\right) / 2 \in \mathcal{P}_{\nu}$. Let $d \in \mathbb{N}, \psi \in C_{0}^{\infty}\left(\mathbb{R}^{d}\right)$ and $|\psi(x)| \leq 1$ for all $x \in \mathbb{R}^{d}$. By condition (B) for every $\varepsilon>0$ there exist a natural number $N \geq d$ and a $C_{b}^{2,1}$-mapping $\left(b_{\varepsilon}^{k}\right)_{k=1}^{N}$ on $\mathbb{R}^{N} \times\left[0, T_{0}\right]$ such that

$$
\int_{0}^{T_{0}} \int_{\mathbb{R}^{\infty}}\left|A_{N}^{-1 / 2}(x, s)\left(B_{N}(x, s)-b_{\varepsilon}\left(x_{1}, \ldots, x_{N}, s\right)\right)\right|^{2} \sigma_{s}(d x) d s<\varepsilon .
$$

Fix $t \in J_{\psi}^{\sigma^{1}} \cap J_{\psi}^{\sigma^{2}} \cap J_{\psi^{2}}^{\sigma^{1}} \cap J_{\psi^{2}}^{\sigma^{2}}$. Let $f$ be a solution to the finite-dimensional Cauchy problem

$$
\left\{\begin{array}{l}
\partial_{t} f+\sum_{i, j=1}^{N} a^{i j} \partial_{x_{i}} \partial_{x_{j}} f+\sum_{i=1}^{N} b_{\varepsilon}^{i} \partial_{x_{i}} f=0 \quad \text { on } \mathbb{R}^{N} \times(0, t), \\
f(t, x)=\psi(x) .
\end{array}\right.
$$


It is known (see, e.g., [31, Theorem 1.3] and also [18,23], and [36]) that a solution exists and belongs to the class $C_{b}\left(\mathbb{R}^{N} \times[0, t]\right) \bigcap C_{b}^{2,1}\left(\mathbb{R}^{N} \times(0, t)\right)$. Moreover, according to the maximum principle $|f(x, s)| \leq 1$ for all $(x, s) \in \mathbb{R}^{N} \times[0, t]$. Set $\mu=\sigma^{1}-\sigma^{2}$. The measure $\mu$ solves the Cauchy problem (1.1) with zero initial condition. Applying Lemma 1.1 and Remark 1.3 with $\varphi=f$, we obtain

$$
\int_{\mathbb{R}^{\infty}} f(x, t) \mu_{t}(d x)=\int_{0}^{t} \int_{\mathbb{R}^{\infty}}\left[\partial_{s} f+\sum_{i, j=1}^{N} a^{i j} \partial_{x_{j}} \partial_{x_{i}} f+\sum_{i=1}^{N} B^{i} \partial_{x_{i}} f\right] d \mu_{s} d s .
$$

Therefore,

$$
\int_{\mathbb{R}^{\infty}} \psi d \mu_{t}=\int_{0}^{t} \int_{\mathbb{R}^{\infty}}\left\langle B-b_{\varepsilon}, \nabla f\right\rangle d \mu_{s} d s .
$$

Let us estimate the following expression:

$$
\int_{0}^{t} \int_{\mathbb{R}^{\infty}}\left|\sqrt{A_{N}} \nabla f\right|^{2} d \sigma_{s} d s
$$

Using (1.2) for $\sigma$ and $\varphi=f^{2}$, taking into account that $\left(\partial_{s}+L\right)\left(f^{2}\right)=2 f\left(\partial_{s}+\right.$ $L) f+2\left|\sqrt{A_{N}} \nabla f\right|^{2}$, and recalling that $t \in J_{\psi^{2}}^{\sigma^{1}} \cap J_{\psi^{2}}^{\sigma^{2}}$, we obtain from (2.1) (again by Remark 1.3) that

$$
\begin{aligned}
& \int_{\mathbb{R}^{\infty}} \psi^{2} d \sigma_{t}-\int_{\mathbb{R}^{\infty}} f^{2}(x, 0) v(d x) \\
& =2 \int_{0}^{t} \int_{\mathbb{R}^{\infty}}\left[\left|\sqrt{A_{N}} \nabla f\right|^{2}+f \sum_{i=1}^{N}\left(B^{i}-b_{\varepsilon}^{i}\right) \partial_{x_{i}} f\right] d \sigma_{s} d s .
\end{aligned}
$$

Therefore,

$$
\begin{aligned}
& \int_{0}^{t} \int_{\mathbb{R}^{\infty}}\left|\sqrt{A_{N}} \nabla f\right|^{2} d \sigma_{s} d s \\
& \leq 2+\int_{0}^{T_{0}} \int_{\mathbb{R}^{\infty}}\left|A_{N}^{-1 / 2}(x, s)\left(B_{N}(x, s)-b_{\varepsilon}\left(x_{1}, \ldots, x_{N}, s\right)\right)\right|^{2} \sigma_{s}(d x) d s .
\end{aligned}
$$

Thus we obtain the estimate

$$
\int_{0}^{t} \int_{\mathbb{R}^{\infty}}\left|\sqrt{A_{N}} \nabla f\right|^{2} d \sigma_{s} d s \leq 2+\varepsilon
$$

Applying (2.2) and (2.3) and the fact that $|\mu| \leq \sigma^{1}+\sigma^{2}=2 \sigma$ we have

$$
\int_{\mathbb{R}^{\infty}} \psi d \mu_{t} \leq 2 \sqrt{\varepsilon(2+\varepsilon)}
$$


Since $\varepsilon>0$ was arbitrary, we obtain

$$
\int_{\mathbb{R}^{\infty}} \psi d \mu_{t} \leq 0 .
$$

Replacing $\psi$ with $-\psi$ we arrive at the equality

$$
\int_{\mathbb{R}^{\infty}} \psi d \mu_{t}=0 .
$$

Therefore,

$$
\int_{\mathbb{R}^{\infty}} \psi d \sigma_{t}^{1}=\int_{\mathbb{R}^{\infty}} \psi d \sigma_{t}^{2}
$$

for every $t \in J_{\psi}^{\sigma^{1}} \cap J_{\psi}^{\sigma^{2}} \cap J_{\psi^{2}}^{\sigma^{1}} \cap J_{\psi^{2}}^{\sigma^{2}}$, hence for almost every $t \in\left[0, T_{0}\right]$. Thus, $\sigma^{1}=\sigma^{2}$.

We now consider a typical example to which the previous theorem applies, namely, the Fokker-Planck-Kolmogorov equations associated with stochastic partial differential equations of reaction diffusion type on a domain $D \subset \mathbb{R}^{d}$, i.e.,

$$
d u(t)=\sigma(u(t), t) d W(t)+B(u(t), t) d t, t \in\left[0, T_{0}\right],
$$

where $\sigma \sigma^{*}=A$ and $u(t) \in L^{2}(D)$. Furthermore, $W(t), t \geq 0$, is a cylindrical Wiener process in $L^{2}(D)$ on a stochastic basis $\left(\Omega, \mathcal{F},\left(\mathcal{F}_{t}\right), \overline{\mathrm{P}}\right)$ and $u(0)$ has the law $v$. Below we denote by $u$ generic elements of functional spaces such as $L^{2}(D)$ which we embed into $\mathbb{R}^{\infty}$ (e.g., by using a suitable orthonormal basis) to be able to apply our framework above.

Example 2.4 (Reaction diffusion equations in dimension $d$ with infinite trace).

Suppose that $D \subset \mathbb{R}^{d}$ is an open bounded set and $\left\{e_{k}\right\}$ is an eigenbasis of the Laplacian on $L^{2}(D)$ with zero boundary condition, i.e., $\Delta e_{k}=-\lambda_{k} e_{k}, \lambda_{k}>0$. Let $f: D \times \mathbb{R} \times\left[0, T_{0}\right] \rightarrow \mathbb{R}$ be a Borel function. Set

$$
B(u, t)(z)=\Delta u(z)+f(z, u(z), t), z \in D,
$$

that is,

$$
B^{i}(u, t)=-\lambda_{i} u_{i}+\left\langle f(\cdot, u(\cdot), t), e_{i}\right\rangle_{2}, u \in L^{2}(D), u_{i}=\left\langle u, e_{i}\right\rangle_{2} .
$$

Assume that the coefficients $a^{i j}$ satisfy (A) with $\gamma_{N}=\gamma>0$ independent of $N$. For instance, the last assumption is true if $a^{i j}=\left\langle S e_{i}, e_{j}\right\rangle_{2}$ for some invertible symmetric positive operator $S$ on $L^{2}(D)$.

Assume also that there exist a Borel function $C \geq 0$ on $\left[0, T_{0}\right]$ and a number $m \geq 1$ such that

$$
|f(z, u, t)| \leq C(t)+C(t)|u|^{m}
$$


Set

$$
L \varphi=\sum_{i, j=1}^{\infty} a^{i j} \partial_{e_{i}} \partial_{e_{j}} \varphi+\sum_{i=1}^{\infty} B^{i} \partial_{e_{i}} \varphi,
$$

where $\partial_{e_{i}}$ denotes the partial derivative along $e_{i}$ (and corresponds to $\partial_{x_{i}}$ if we embed $L^{2}(D)$ into $\mathbb{R}^{\infty}$ by the mapping $\left.u \mapsto\left(\left\langle u, e_{i}\right\rangle_{2}\right)\right)$. Then there is at most one probability solution $\mu=\mu_{t}(d u) d t$, i.e., $\mu_{t} \geq 0$ and $\mu_{t}\left(\mathbb{R}^{\infty}\right)=1$ for every $t \in\left(0, T_{0}\right)$, to the Cauchy problem (1.1) such that

$$
\int_{0}^{T_{0}}\left(1+C(t)^{2}\right) \int_{L^{2}(D)}\|u\|_{2 m}^{2 m} \mu_{t}(d u) d t<\infty .
$$

Proof. The mapping $u \mapsto\left(u_{i}\right)$ defines an embedding $L^{2}(D) \rightarrow \mathbb{R}^{\infty}$. Extending $B^{i}$ and $a^{i j}$ to all of $\mathbb{R}^{\infty} \times\left[0, T_{0}\right]$ by zero we end up in the framework described above. Set

$$
F^{i}(u, t)=\left\langle f(\cdot, u(\cdot), t), e_{i}\right\rangle_{2}
$$

Note that

$$
\sum_{i=1}^{\infty}\left|F^{i}(u, t)\right|^{2}=\|f(\cdot, u(\cdot), t)\|_{L^{2}}^{2} \leq C(t)^{2}+C(t)^{2}\|u\|_{2 m}^{2 m} .
$$

Thus we have $B^{i}=A^{i}+F^{i}$, where $A^{i}(u)=-\lambda_{i} u_{i}$ and $\|F\|_{l^{2}} \in L^{2}(\mu)$, and Example 2.1(iii) applies with $\alpha_{k}=1$.

Let now $d=1, D=(0,1)$ and $\Delta=\frac{d^{2}}{d z^{2}}$. We recall that according to [7] and [8] if $a^{i j}=\alpha \delta^{i j}$ with $\alpha>0$ and if

$$
f(z, u, t)=f_{1}(z, u, t)+f_{2}(z, u, t),
$$

where $(u, t) \mapsto f_{i}(z, u, t)$ are continuous for each $z$ and for some nonnegative functions $c_{1}, c_{3} \in L^{2}\left[0, T_{0}\right], c_{2} \in L^{1}\left[0, T_{0}\right]$ and all $t, z, u$ we have

(i) $\left|f_{1}(z, u, t)\right| \leq c_{1}(t)\left(1+|u|^{m}\right)$,

(ii) $\left(f_{1}(z, u, t)-f_{1}(z, v, t)\right)(u-v) \leq c_{2}(t)|u-v|^{2}$,

(iii) $\left|f_{2}(z, u, t)\right| \leq c_{3}(t)(1+|u|)$,

then for every initial value $v$ with $\|u\|_{2 m}^{2 m} \in L^{1}(v)$ there exists a probability solution $\mu$ of the Cauchy problem (1.1) such that $\left(1+c_{1}(t)+c_{3}(t)\right)^{2}\left(1+\|u\|_{2 m}^{2 m}\right) \in L^{1}(\mu)$. It follows from the previous example that such a solution is unique, which improves the uniqueness result from [7] and [8] (where the diffusion operator was invertible).

We now present another uniqueness condition that applies to degenerate (even zero) diffusion matrices. Let us list our new assumptions $\left(\mathrm{A}^{\prime}\right)$ and $\left(\mathrm{B}^{\prime}\right)$.

$\left(\mathrm{A}^{\prime}\right) A(x, t)=\left(a^{i j}(x, t)\right)$, where each function $a^{i j}$ is bounded and depends only on the variables $x_{1}, x_{2}, \ldots, x_{\max \{i, j\}}, t$ and for every natural number $N$ the matrix 
$A_{N}$ is symmetric nonnegative and the elements $\sigma_{N}^{i j}$ of the matrix $\sigma_{N}:=\sqrt{A_{N}}$ are in the class $C^{\infty}\left(\mathbb{R}^{N} \times\left[0, T_{0}\right]\right)$.

Let $v$ be a Borel probability measure on $\mathbb{R}^{\infty}$ and let $\mathbb{P}_{v}$ be some convex set of probability solutions $\mu=\mu_{t}(d x) d t$ of (1.1), i.e., $\mu_{t} \geq 0$ and $\mu_{t}\left(\mathbb{R}^{\infty}\right)=1$ for every $t \in\left(0, T_{0}\right)$, such that $\left|B^{k}\right| \in L^{1}(\mu)$ for each $k \in \mathbb{N}$ and the following condition holds:

(B') for every $\varepsilon>0$ and every natural number $d$ there exist a natural number $N=N(\varepsilon, d) \geq d$, a $C^{\infty}$-mapping $b_{\varepsilon}=\left(b_{\varepsilon}^{k}\right)_{k=1}^{N}: \mathbb{R}^{N} \times\left[0, T_{0}\right] \rightarrow \mathbb{R}^{N}$, a function $\theta_{\varepsilon}$ on $\mathbb{R}^{N}$, a function $V_{\varepsilon} \in C^{2}\left(\mathbb{R}^{N}\right)$ with $V_{\varepsilon} \geq 1$, and numbers $C_{\varepsilon} \geq 0$ and $\delta_{\varepsilon}>0$ such that

(i) $\sqrt{V_{\varepsilon}\left(P_{N} x\right)},\left|B_{N}(x, t)-b_{\varepsilon}\left(P_{N} x, t\right)\right| \sqrt{V_{\varepsilon}\left(P_{N} x\right)} \in L^{1}(\mu)$ and

$$
\int_{0}^{T_{0}} \int_{\mathbb{R}^{\infty}}\left|B_{N}(x, t)-b_{\varepsilon}\left(P_{N} x, t\right)\right| \sqrt{V_{\varepsilon}\left(P_{N} x\right)} e^{C_{\varepsilon}\left(T_{0}-t\right) / 2} \mu_{t}(d x) d t<\varepsilon,
$$

where $B_{N}=\left(B^{1}, \ldots, B^{N}\right)$;

(ii) the matrix $\mathcal{B}=\left(\partial_{x_{j}} b_{\varepsilon}^{i}\right)_{i, j \leq N}$ and the operator

$$
L_{A_{N}, b_{\varepsilon}} \varphi(x, t)=\sum_{i, j \leq N} a^{i j}(x, t) \partial_{x_{i}} \partial_{x_{j}} \varphi(x, t)+\sum_{i \leq N} b_{\varepsilon}^{i}(x, t) \partial_{x_{i}} \varphi(x, t)
$$

satisfy the estimates

$$
\langle\mathcal{B}(x, t) h, h\rangle \leq \theta_{\varepsilon}(x)|h|^{2} \forall h \in \mathbb{R}^{N}, \quad L_{A_{N}, b_{\varepsilon}} V_{\varepsilon}(x, t) \leq\left(C_{\varepsilon}-\Lambda(x, t)\right) V_{\varepsilon}(x),
$$

where

$$
\left.\Lambda(x, t):=4 \sum_{i, j, k \leq N}\left|\partial_{x_{k}} \sigma_{N}^{i j}(x, t)\right|^{2}+2 \theta_{\varepsilon}(x)+\delta_{\varepsilon}\left(1+|x|^{2}\right)^{-1}\left|b_{\varepsilon}(x, t)\right|^{2}\right)
$$

for every $(x, t) \in \mathbb{R}^{N} \times\left[0, T_{0}\right]$.

In the notation for $N$ we omit indication of its dependence on $d$ and $\varepsilon$; in the notation for $b_{\varepsilon}, \theta_{\varepsilon}, V_{\varepsilon}, \delta_{\varepsilon}$ also the indication of dependence on $d$ is omitted. Recall also that $|\cdot|$ is the standard Euclidean norm.

Theorem 2.5. If $\left(\mathrm{A}^{\prime}\right)$ and $\left(\mathrm{B}^{\prime}\right)$ hold, then the set $\mathbb{P}_{v}$ contains at most one element.

Remark 2.6. The above condition takes a simpler form in the special, but very important case of $A=0$.

In the case $A=0$ the above assumptions $\left(\mathrm{B}^{\prime}\right)$ can be reformulated in the following way:

- there exist natural numbers $N_{k}$ with $N_{k}<N_{k+1}$, positive numbers $\delta_{k}$ and $C_{k}$, a vector field $b_{k} \in C^{\infty}\left(\mathbb{R}^{N_{k}} \times[0, T]\right)$ and a positive function $V_{k} \in C^{2}\left(\mathbb{R}^{N_{k}}\right)$ such that 
(i) $\sqrt{V_{k}},\left|b_{k}-B\right| \sqrt{V_{k}} \in L^{1}(\mu)$ and

$$
\lim _{k \rightarrow \infty} \int_{0}^{T} \int_{\mathbb{R}^{\infty}}\left|b_{k}\left(P_{N_{k}} x, t\right)-B_{N_{k}}(x, t)\right| \sqrt{V_{k}\left(P_{N_{k}} x\right)} e^{C_{k}(T-t) / 2} \mu_{t}(d x) d t=0,
$$

(ii) $V_{k}(x) \geq 1$ and

$$
\left\langle b_{k}(x, t), \nabla V_{k}(x)\right\rangle \leq\left(C_{k}-\delta_{k}\left(1+|x|^{2}\right)^{-1}\left|b_{k}(x, t)\right|^{2}-2 \max _{|h|=1}\left\langle\mathcal{B}_{k}(x, t) h, h\right\rangle\right) V_{k}(x)
$$

for every $k$ and $(x, t) \in \mathbb{R}^{N_{k}} \times[0, T]$.

Note that in typical examples

$$
N_{k}=k, \quad b_{k}(x, t)=B_{k}\left(P_{k} x, t\right), \quad V_{k}(x)=V\left(P_{k} x\right),
$$

where

$$
V(x)=\exp \left(\kappa\|x\|_{l^{2}}^{2}\right) \quad \text { or } \quad V(x)=\exp \left(\kappa\|x\|_{l_{\lambda}^{2}}^{2}\right) \text {. }
$$

\section{Remark 2.7.}

(i) If $A=\left(a^{i j}\right)$ is a constant matrix and $\left|b_{\varepsilon}(x, t)\right| \leq C_{1}(N)+C_{1}(N)|x|$, then the estimate $L_{A_{N}, b_{\varepsilon}} V \leq\left(C_{\varepsilon}-\Lambda\right) V_{\varepsilon}$ in condition $\left(\mathrm{B}^{\prime}\right)($ ii) is fulfilled if

$$
L_{A_{N}, b_{\varepsilon}} V_{\varepsilon}(x, t) \leq\left(C_{\varepsilon}-2 \theta_{\varepsilon}(x)\right) V_{\varepsilon}(x) \quad \forall(x, t) \in \mathbb{R}^{N} \times\left[0, T_{0}\right]
$$

(ii) If $A=\left(a^{i j}\right)$ is a constant matrix and $\left|b_{\varepsilon}(x, t)\right| \leq C_{1}(N)+C_{1}(N)|x|^{2}$, then the estimate $L_{A_{N}, b_{\varepsilon}} V \leq\left(C_{\varepsilon}-\Lambda\right) V_{\varepsilon}$ in condition (B')(ii) is fulfilled if

$$
L_{A_{N}, b_{\varepsilon}} V_{\varepsilon}(x, t) \leq\left(C_{\varepsilon}-2 \theta_{\varepsilon}(x)-\delta_{\varepsilon}|x|^{2}\right) V_{\varepsilon}(x)
$$

for every $(x, t) \in \mathbb{R}^{N} \times\left[0, T_{0}\right]$ and some $\delta_{\varepsilon}>0$.

(iii) Let $a^{i j}=0$ if $i \neq j$ and $a^{i i}(x, t)=\alpha^{i}\left(x_{1}, x_{2}, \ldots, x_{i}, t\right) \geq 0$. Suppose also that we have $\left|b_{\varepsilon}(x, t)\right| \leq C_{1}(N)+C_{1}(N)|x|^{2}$. Then the estimate $L_{A_{N}, b_{\varepsilon}} V \leq$ $\left(C_{\varepsilon}-\Lambda\right) V_{\varepsilon}$ in condition $\left(\mathrm{B}^{\prime}\right)($ ii) is fulfilled if

$$
\begin{aligned}
& L_{A_{N}, b_{\varepsilon}} V_{\varepsilon}(x, t) \leq\left(C_{\varepsilon}-\Lambda(x, t)\right) V_{\varepsilon}(x), \\
& \Lambda(x, t):=4 \sum_{i \leq N} \sum_{k \leq i} \frac{\left|\partial_{x_{k}} \alpha^{i}(x, t)\right|^{2}}{\alpha^{i}(x, t)}+2 \theta_{\varepsilon}(x)+\delta_{\varepsilon}|x|^{2} .
\end{aligned}
$$

(iv) We note that $\left(\mathrm{B}^{\prime}\right)$ is a substantial generalization of a corresponding condition in [32] (see Section 4 there, where exponents of quadratic forms are used for $V$ along with some additional restrictions). 
Let us illustrate condition ( $\left.\mathrm{B}^{\prime}\right)$. In (i)-(iii) in the next example we discuss condition $\left(\mathrm{B}^{\prime}\right)(\mathrm{ii})$ assuming that $\left(\mathrm{B}^{\prime}\right)(\mathrm{i})$ is already satisfied for some $b_{\varepsilon}$ and only in (iv) we discuss how both (i) and (ii) can be ensured. Moreover, we deal with $V(x)=$ $\exp \left(\kappa\|x\|_{l^{2}}^{2}\right)$ or $V(x)=\exp \left(\kappa\|x\|_{l_{\lambda}^{2}}^{2}\right)$ and $b_{\varepsilon}^{i}(x)=-\lambda_{i} x_{i}+f^{i}(x, t)$. In particular, such $V$ actually does not depend on $\varepsilon$ in the sense that, for any $N$, on $\mathbb{R}^{N}$ we use the restriction of a common function on $\mathbb{R}^{\infty}$.

Example 2.8. We assume here that $A=\left(a^{i j}\right)_{i, j \geq 1}$ is a constant matrix, $A_{N}:=$ $\left(a^{i j}\right)_{i, j \leq N}$ is symmetric nonnegative.

(i) Let $b_{\varepsilon}^{k}(x, t)=-\lambda_{k} x_{k}+f^{k}(x, t), x \in \mathbb{R}^{N}$. Then the estimate $\langle\mathcal{B} h, h\rangle \leq$ $\theta_{\varepsilon}(x)|h|^{2}, x, h \in \mathbb{R}^{N}$, follows from the estimate

$$
\langle\mathcal{F}(x, t) h, h\rangle \leq \theta_{\varepsilon}(x)|h|^{2}+\|h\|_{l_{\lambda}^{2}}^{2}, \quad x, h \in \mathbb{R}^{N}, \quad \mathcal{F}=\left(\partial_{x_{j}} f^{i}\right)_{i, j \leq N} .
$$

(ii) Set $V(x)=\exp \left(\kappa \sum_{k=1}^{\infty} x_{k}^{2}\right)$, where $\kappa>0$. Then the inequality $L_{A_{N}, b_{\varepsilon}} V_{\varepsilon} \leq$ $\left(C_{\varepsilon}-\Lambda\right) V_{\varepsilon}$ in (B')(ii) can be written as follows: for some numbers $C_{\varepsilon}$ and $\delta_{\varepsilon}>0$ (dependent on $\varepsilon$ and $d$ ) one has

$$
\begin{aligned}
& \theta_{\varepsilon}(x) \leq C_{\varepsilon}-\kappa\left(\operatorname{tr} A_{N}+2 \kappa\left\langle A_{N} x, x\right\rangle+\left\langle b_{\varepsilon}(x, t), x\right\rangle\right) \\
& -2^{-1} \delta_{\varepsilon}\left(1+|x|^{2}\right)^{-1}\left|b_{\varepsilon}(x, t)\right|^{2}, \quad x \in \mathbb{R}^{N} .
\end{aligned}
$$

Let us consider a more specific case:

$$
b_{\varepsilon}^{k}(x, t)=-\lambda_{k} x_{k}+f^{k}(x, t), \quad f(x, t)=\left(f^{k}(x, t)\right)_{k=1}^{N}, \quad\langle f(x, t), x\rangle \leq 0
$$

and $\left|f^{k}(x, t)\right| \leq C_{1}+C_{2}|x|^{2}$, where $x \in \mathbb{R}^{N}$. Assume that for some $\varepsilon_{0}>0$ and every $N \geq 1$ one has

$$
\varepsilon_{0}\left(\left\langle A_{N} x, x\right\rangle+|x|^{2}\right) \leq\|x\|_{l_{\lambda}^{2}}^{2}, \quad x \in \mathbb{R}^{N} .
$$

Then condition $\left(\mathrm{B}^{\prime}\right)(\mathrm{ii})$ can be rewritten in the following form:

$$
\begin{gathered}
\langle\mathcal{F}(x, t) h, h\rangle \leq \theta_{\varepsilon}(x)|h|^{2}+\|h\|_{l_{\lambda}^{2}}^{2}, \quad x, h \in \mathbb{R}^{N}, \quad \mathcal{F}=\left(\partial_{x_{j}} f^{i}\right)_{i, j \leq N}, \\
\theta_{\varepsilon}(x) \leq C_{\varepsilon}-\kappa \operatorname{tr} A_{N}+2^{-1} \kappa\left(\varepsilon_{0}-\kappa\right)\|x\|_{l_{\lambda}^{2}}^{2}, \quad x \in \mathbb{R}^{N} .
\end{gathered}
$$

Note that in this case we take $V$ with $\kappa<\varepsilon_{0} / 4$.

This assertion follows from (2.4) if we choose $\delta_{\varepsilon}>0$ such that

$$
\delta_{\varepsilon}\left(1+|x|^{2}\right)^{-1}\left|b_{\varepsilon}(x, t)\right|^{2} \leq \varepsilon_{0} \kappa|x|^{2}+1 .
$$


(iii) Let $V(x)=\exp \left(\kappa\|x\|_{l_{\lambda}^{2}}^{2}\right)=\exp \left(\kappa \sum_{n=1}^{\infty} \lambda_{n} x_{n}^{2}\right)$. Then the inequality $L_{A_{N}, b_{\varepsilon}} V_{\varepsilon} \leq\left(C_{\varepsilon}-\Lambda\right) V_{\varepsilon}$ in $\left(\mathrm{B}^{\prime}\right)(\mathrm{ii})$ can be written as follows: for some constants $C_{\varepsilon}$ and $\delta_{\varepsilon}>0$ one has

$$
\begin{aligned}
\theta_{\varepsilon}(x) \leq C_{\varepsilon} & -\kappa\left(\sum_{i=1}^{N} a^{i i} \lambda_{i}+2 \kappa \sum_{i, j \leq N} a^{i j} \lambda_{i} \lambda_{j} x_{i} x_{j}+\sum_{i=1}^{N} \lambda_{i} b_{\varepsilon}^{i}(x, t) x_{i}\right) \\
- & 2^{-1} \delta_{\varepsilon}\left(1+|x|^{2}\right)^{-1}\left|b_{\varepsilon}(x, t)\right|^{2}, \quad x \in \mathbb{R}^{N} .
\end{aligned}
$$

Let us consider again a more specific case:

$$
\begin{aligned}
& b_{\varepsilon}^{k}(x, t)=-\lambda_{k} x_{k}+f^{k}(x, t), \quad f(x, t)=\left(f^{k}(x, t)\right)_{i=1}^{N}, \\
& \langle f(x, t), x\rangle_{l_{\lambda}^{2}}=\sum_{i=1}^{N} \lambda_{i} f^{i}(x, t) x_{i} \leq 0
\end{aligned}
$$

and $\left|f^{k}(x, t)\right| \leq C_{1}+C_{2}|x|^{2}$, where $x \in \mathbb{R}^{N}$. Assume that for some $\varepsilon_{0}>0$ and every $N \geq 1$ one has

$$
\varepsilon_{0} \sum_{i, j \leq N} a^{i j} \lambda_{i} \lambda_{j} x_{i} x_{j}+\varepsilon_{0}|x|^{2} \leq \sum_{i \leq N} \lambda_{i}^{2} x_{i}^{2} .
$$

Then condition $\left(\mathrm{B}^{\prime}\right)(\mathrm{ii})$ can be rewritten in the following form:

$$
\begin{gathered}
\langle\mathcal{F}(x, t) h, h\rangle \leq \theta_{\varepsilon}(x)|h|^{2}+\sum_{i=1}^{N} \lambda_{i} h_{i}^{2}, \quad x, h \in \mathbb{R}^{N}, \quad \mathcal{F}=\left(\partial_{x_{j}} f^{i}\right)_{i, j \leq N} \\
\theta_{\varepsilon}(x) \leq C_{\varepsilon}-\kappa \sum_{i=1}^{N} a^{i i} \lambda_{i}+2^{-1} \kappa\left(\varepsilon_{0}-\kappa\right) \sum_{i=1}^{N} \lambda_{i}^{2} x_{i}^{2}, \quad x \in \mathbb{R}^{N} .
\end{gathered}
$$

Note that in this case we take $V(x)$ with $\kappa<\varepsilon_{0} / 4$.

This assertion follows from (2.5) if we choose $\delta_{\varepsilon}>0$ such that

$$
\delta_{\varepsilon}\left(1+|x|^{2}\right)^{-1}\left|b_{\varepsilon}(x, t)\right|^{2} \leq \varepsilon_{0} \kappa|x|^{2}+1, \quad x \in \mathbb{R}^{N} .
$$

(iv) Let consider a yet more special case, which, however, will be useful below. Namely, suppose that $a^{i j}=0$ if $i \neq j, a^{i i}=\alpha_{i}>0$ are constant and the restrictions of the functions $B^{k}$ to the spaces $\mathbb{R}^{n}$ are infinitely differentiable. Let $\left|B^{k}\right| \in L^{2}(\mu)$ for all $k \in \mathbb{N}$. Suppose also that there are a $\mu$-integrable function $V \geq 1$ on $\mathbb{R}^{\infty}$ with smooth restrictions to $\mathbb{R}^{n}$ and a number $C_{0} \geq 0$ such that, for every $\varepsilon>0$ and $d \in \mathbb{N}$ one can find numbers $N \geq d$ and $\delta_{\varepsilon}>0$ such that

$$
\int_{0}^{T_{0}} \int_{\mathbb{R}^{\infty}}\left|B_{N}(x, t)-B_{N}\left(P_{N} x, t\right)\right| \sqrt{V\left(P_{N} x\right)} \mu_{t}(d x) d t<\varepsilon
$$


where $B_{N}=\left(B^{1}, \ldots, B^{N}\right)$, i.e. we take for $b_{\varepsilon}$ the restriction of $B_{N}$ to $\mathbb{R}^{N}$ with a sufficiently large $N$, and, in addition, the matrix $\mathcal{B}=\left(\partial_{x_{j}} B^{i}\right)_{i, j \leq N}$ and the operator

$$
L_{N} \varphi(x, t)=\sum_{i \leq N}\left[\alpha_{i} \partial_{x_{i}} \partial_{x_{j}} \varphi(x, t)+B^{i}(x, t) \partial_{x_{i}} \varphi(x, t)\right], \quad x \in \mathbb{R}^{N},
$$

satisfy the estimate

$$
L_{N} V(x, t) \leq\left(C_{0}-\Lambda(x, t)\right) V(x),(x, t) \in \mathbb{R}^{N} \times\left[0, T_{0}\right],
$$

where

$$
\Lambda(x, t):=\delta_{\varepsilon}\left(1+|x|^{2}\right)^{-1}\left|B_{N}(x, t)\right|^{2}+2 \sup _{|h| \leq 1}\langle\mathcal{B}(x, t) h, h\rangle .
$$

Then condition $\left(\mathrm{B}^{\prime}\right)$ (ii) is fulfilled. We shall apply this case with the function $V(x)=\exp \left(\kappa \sum_{n=1}^{\infty} \lambda_{n} x_{n}^{2}\right)$. Finally, in order to ensure also (i) in ( $\left.\mathrm{B}^{\prime}\right)$, it suffices to have an integrable majorant for the functions $\left|B_{N}(x, t)-B_{N}\left(P_{N} x, t\right)\right| \sqrt{V\left(P_{N} x\right)}$ and convergence $\left|B_{N}(x, t)-B_{N}\left(P_{N} x, t\right)\right| \rightarrow 0$ almost everywhere.

For the proof of Theorem 2.5 we need the following lemma. In its formulation and proof for notational simplicity we omit indication of $\varepsilon$ for $b, \theta$ and $\delta$.

Let $\eta \in C_{0}^{\infty}\left(\mathbb{R}^{1}\right)$ be such that $\eta(x)=1$ if $|x| \leq 1$ and $\eta(x)=0$ if $|x|>2$, $0 \leq \eta \leq 1$ and there exists a number $C_{\eta}>0$ such that $\left|\eta^{\prime}(x)\right|^{2} \eta^{-1}(x) \leq C_{\eta}$ for every $x$.

Lemma 2.9. Let $b \in C^{\infty}\left(\mathbb{R}^{N}, \mathbb{R}^{N}\right)$. Assume that there exist a function $\theta$ on $\mathbb{R}^{N}$, a function $V \in C^{2}\left(\mathbb{R}^{N}\right)$ with $V \geq 1$, and numbers $C_{0} \geq 0$ and $\delta>0$ such that for all $(x, t) \in \mathbb{R}^{N} \times\left[0, T_{0}\right], h \in \mathbb{R}^{N}$ one has

$$
\begin{gathered}
\langle\mathcal{B}(x, t) h, h\rangle \leq \theta(x)|h|^{2}, \quad \mathcal{B}=\left(\partial_{x_{j}} b^{i}\right)_{i, j \leq N}, \\
L_{A_{N}, b} V(x, t) \leq\left(C_{0}-\Lambda(x, t)\right) V(x), \\
\Lambda(x, t):=4 \sum_{i, j, k \leq N}\left|\partial_{x_{k}} \sigma_{N}^{i j}(x, t)\right|^{2}+2 \theta(x)+\delta\left(1+|x|^{2}\right)^{-1}|b(x, t)|^{2} .
\end{gathered}
$$

Then there exists a number $\kappa>0$ dependent on $\delta, \operatorname{tr} A, C_{\eta}$ such that for every $M>0$ and every $s \in\left(0, T_{0}\right)$ the Cauchy problem

$$
\partial_{t} f+\zeta_{M} L_{A_{N}, b} f=0,\left.\quad f\right|_{t=s}=\psi,
$$

where $\psi \in C_{b}^{\infty}\left(\mathbb{R}^{N}\right), \zeta_{M}(x)=\eta\left(\left(1+|x|^{2}\right)^{\kappa} / M\right)$, has a smooth solution $f$ such that

$$
|f(x, t)| \leq \max _{x}|\psi(x)|, \quad|\nabla f(x, t)|^{2} \leq e^{\left(C_{0}+1\right)(s-t)} V(x) \max _{x}|\nabla \psi(x)|^{2} / 2 .
$$


Proof. The existence of a smooth bounded (with bounded derivatives) solution $f$ is well known (see [30, Theorem 2], [36, Theorem 3.2.4, Theorem 3.2.6]). The maximum principle implies that $|f(x, t)| \leq \max _{x}|\psi(x)|$. Set

$$
u=2^{-1} \sum_{k=1}^{N}\left|\partial_{x_{k}} f\right|^{2} .
$$

Differentiating the equation $\partial_{t} f+\zeta_{M} L_{A_{N}, b} f=0$ with respect to $x_{k}$ and multiplying by $\partial_{x_{k}} f$, we obtain

$$
\begin{aligned}
\partial_{t} u & +\zeta_{M} L_{A_{N}, b} u+\zeta_{M}\langle\mathcal{B} \nabla f, \nabla f\rangle+\left\langle\nabla \zeta_{M}, \nabla f\right\rangle\langle b, \nabla f\rangle \\
& +\zeta_{M} \partial_{x_{k}} a^{i j} \partial_{x_{i} x_{j}} f \partial_{x_{k}} f \\
& +a^{i j} \partial_{x_{i} x_{j}} f \partial_{x_{k}} f \partial_{x_{k}} \zeta_{M}-\zeta_{M} a^{i j} \partial_{x_{k} x_{i}} f \partial_{x_{k} x_{j}} f=0 .
\end{aligned}
$$

Note that

$$
\langle\mathcal{B} \nabla f, \nabla f\rangle \leq 2 \theta u, \quad\left\langle\nabla \zeta_{M}, \nabla f\right\rangle\langle b, \nabla f\rangle \leq 2\left|\nabla \zeta_{M}\right||b| u .
$$

Let us consider the expression

$$
\zeta_{M} \partial_{x_{k}} a^{i j} \partial_{x_{i} x_{j}} f \partial_{x_{k}} f+a^{i j} \partial_{x_{i} x_{j}} f \partial_{x_{k}} f \partial_{x_{k}} \zeta_{M}-\zeta_{M} a^{i j} \partial_{x_{k} x_{i}} f \partial_{x_{k} x_{j}} f .
$$

Recall that $A_{N}=\sigma_{N}^{2}$. We have

$$
\begin{aligned}
\sum_{i, j, k} \partial_{x_{k}} a^{i j} \partial_{x_{i} x_{j}} f \partial_{x_{k}} f & =2 \sum_{i, j, m, k} \partial_{x_{k}} \sigma_{N}^{i m} \sigma^{m j} \partial_{x_{i} x_{j}} f \partial_{x_{k}} f \\
& \leq 2 \sum_{i, m}\left(\sum_{k}\left|\partial_{x_{k}} \sigma_{N}^{i m}\right|^{2}\right)^{1 / 2}\left(\sum_{k}\left|\partial_{x_{k}} f\right|^{2}\right)^{1 / 2}\left|\sum_{j} \sigma_{N}^{m j} \partial_{x_{i} x_{j}} f\right|,
\end{aligned}
$$

which is estimated by

$$
4 u \sum_{i, m, k}\left|\partial_{x_{k}} \sigma_{N}^{i m}\right|^{2}+4^{-1} \sum_{i, m}\left|\sum_{j} \sigma_{N}^{m j} \partial_{x_{i} x_{j}} f\right|^{2} .
$$

Note that

$$
\sum_{i, m}\left|\sum_{j} \sigma_{N}^{m j} \partial_{x_{i} x_{j}} f\right|^{2}=\sum_{i, j, k} a^{i j} \partial_{x_{k} x_{j}} f \partial_{x_{k} x_{j}} f
$$


which is the square of the Hilbert-Schmidt norm of the operator $\sigma_{N} D^{2} f$ (recall again that $A_{N}=\sigma_{N}^{2}$ ). Thus,

$$
\zeta_{M} \partial_{x_{k}} a^{i j} \partial_{x_{i} x_{j}} f \partial_{x_{k}} f \leq 4 \zeta_{M} u \sum_{i, m, k}\left|\partial_{x_{k}} \sigma^{i m}\right|^{2}+4^{-1} \zeta_{M} \sum_{i, j, k} a^{i j} \partial_{x_{k} x_{j}} f \partial_{x_{k} x_{i}} f
$$

We now estimate the term

$$
a^{i j} \partial_{x_{i} x_{j}} f \partial_{x_{k}} f \partial_{x_{k}} \zeta_{M}
$$

Applying the inequality $x y \leq(4+4 \operatorname{tr} A)^{-1} x^{2}+(1+\operatorname{tr} A) y^{2}$ we obtain

$$
a^{i j} \partial_{x_{i} x_{j}} f \partial_{x_{k}} f \partial_{x_{k}} \zeta_{M} \leq 2 u \frac{\left|\nabla \zeta_{M}\right|^{2}}{\zeta_{M}}(1+\operatorname{tr} A)+\zeta_{M}(4+4 \operatorname{tr} A)^{-1}\left(a^{i j} \partial_{x_{i} x_{j}} f\right)^{2} .
$$

Note also that the following inequality is true:

$$
\left(\sum_{i, j} a^{i j} \partial_{x_{i} x_{j}} f\right)^{2} \leq\left(\sum_{i} a^{i i}\right)\left(\sum_{i, j, k} a^{i j} \partial_{x_{i} x_{k}} f \partial_{x_{j} x_{k}} f\right) .
$$

This follows by the inequality

$$
|\operatorname{tr}(A B)|^{2} \leq \operatorname{tr} A \operatorname{tr}\left(A B^{2}\right)
$$

valid for symmetric matrices $A$ and $B$, where $A$ is nonnegative. The latter is due to the Cauchy inequality applied to the inner product $\langle X, Y\rangle=\operatorname{tr}\left(X Y^{*}\right)$ on the space of $N \times N$-matrices and the matrices $X=A^{1 / 2}, Y=B A^{1 / 2}$, for which

$$
\operatorname{tr}\left(Y Y^{*}\right)=\operatorname{tr}\left(B A^{1 / 2} A^{1 / 2} B\right)=\operatorname{tr}\left(A B^{2}\right) .
$$

Therefore, we have the estimate

$$
a^{i j} \partial_{x_{i} x_{j}} f \partial_{x_{k}} f \partial_{x_{k}} \zeta_{M} \leq 2 u \frac{\left|\nabla \zeta_{M}\right|^{2}}{\zeta_{M}}(1+\operatorname{tr} A)+4^{-1} \zeta_{M} \sum_{i, j, k} a^{i j} \partial_{x_{k} x_{j}} f \partial_{x_{k} x_{i}} f .
$$

Applying the above inequalities we estimate (2.8) by

$$
u\left(4 \zeta_{M} \sum_{i, m, k}\left|\partial_{x_{k}} \sigma^{i m}\right|^{2}+2 \frac{\left|\nabla \zeta_{M}\right|^{2}}{\zeta_{M}}(1+\operatorname{tr} A)\right)
$$

Therefore, using (2.7) we arrive at the estimate

$$
\partial_{t} u+\zeta_{M} L_{A_{N}, b} u+Q u \geq 0,
$$

where

$$
Q=2 \frac{\left|\nabla \zeta_{M}\right|^{2}}{\zeta_{M}}(1+\operatorname{tr} A)+\left|\nabla \zeta_{M}\right||b|+2 \zeta_{M} \theta+4 \zeta_{M} \sum_{i, j, k \leq N}\left|\partial_{x_{k}} \sigma_{N}^{i j}\right|^{2}
$$


We have

$$
\left|\nabla \zeta_{M}(x)\right| \leq 4 \kappa\left(1+|x|^{2}\right)^{-1 / 2}\left|\eta^{\prime}\left(\left(1+|x|^{2}\right)^{\kappa} / M\right)\right|
$$

Hence

$$
Q \leq 8 \kappa^{2} C_{\eta}(1+\operatorname{tr} A)+16 \kappa C_{\eta}+\zeta_{M}\left(4 \sum_{i, j, k \leq N}\left|\partial_{x_{k}} \sigma_{N}^{i j}\right|^{2}+2 \theta+2 \kappa\left(1+|x|^{2}\right)^{-1}|b|^{2}\right) .
$$

Let us choose $\kappa>0$ such that

$$
Q \leq 1+\zeta_{M}\left(4 \sum_{i, j, k \leq N}\left|\partial_{x_{k}} \sigma_{N}^{i j}\right|^{2}+2 \theta+\delta\left(1+|x|^{2}\right)^{-1}|b|^{2}\right)
$$

Let us set $u=w V$. Then $w$ satisfies the inequality

$$
\partial_{t} w+\zeta_{M} L_{A_{N}, \widetilde{b}} w+\widetilde{Q} w \geq 0
$$

where

$$
\widetilde{b}^{k}=b^{k}+2 \frac{a^{k j} \partial_{x_{j}} V}{V}, \quad \widetilde{Q}=Q+\zeta_{M} \frac{L_{A_{N}, b} V}{V} .
$$

By our assumptions we have $\widetilde{Q} \leq C_{0}+1$. Since $u(x, s)=|\nabla f(x, s)|^{2} / 2=$ $|\nabla \psi(x)|^{2} / 2$, we have

$$
w(x, s)=V(x)^{-1}|\nabla \psi(x)|^{2} / 2 \leq|\nabla \psi(x)|^{2} / 2 .
$$

Applying the maximum principle (see [36, Theorem 3.1.1]) we obtain

$$
\max _{x}|w(x, t)| \leq e^{\left(C_{0}+1\right)(s-t)} \max _{x}|\nabla \psi(x)|^{2} / 2,
$$

which completes the proof.

We can now prove Theorem 2.5.

Proof. Assume that $\sigma^{1}=\sigma_{t}^{1} d t$ and $\sigma^{2}=\sigma_{t}^{2} d t$ belong to $\mathbb{P}_{\nu}$. By our assumption about $\mathbb{P}_{\nu}$ we have $\sigma=\left(\sigma^{1}+\sigma^{2}\right) / 2 \in \mathbb{P}_{\nu}$. Let $d \in \mathbb{N}, \psi \in C_{0}^{\infty}\left(\mathbb{R}^{d}\right)$ and $|\nabla \psi(x)|+$ $|\psi(x)| \leq 1$ for all $x \in \mathbb{R}^{d}$. For every $\varepsilon>0$ and every natural number $d$ we find a natural number $N \geq d$, a $C^{\infty}$-mapping $b_{\varepsilon}=\left(b_{\varepsilon}^{k}\right)_{k=1}^{N}: \mathbb{R}^{N} \times\left[0, T_{0}\right] \rightarrow \mathbb{R}^{N}$, a function $\theta_{\varepsilon}$ on $\mathbb{R}^{N}$, a function $V_{\varepsilon} \in C^{2}\left(\mathbb{R}^{N}\right), V \geq 1$, and numbers $C_{\varepsilon} \geq 0$ and $\delta_{\varepsilon}>0$ such that (i) and (ii) in condition ( $\left.\mathrm{B}^{\prime}\right)$ are fulfilled.

Let a function $\eta \in C_{0}^{\infty}\left(\mathbb{R}^{1}\right)$ be such that $\eta(x)=1$ if $|x| \leq 1$ and $\eta(x)=0$ if $|x|>2,0 \leq \eta \leq 1$ and there exists a number $C_{\eta}>0$ such that $\left|\eta^{\prime}(x)\right|^{2} \eta^{-1}(x) \leq$ $C_{\eta}$ for every $x$. Let $\kappa>0$ be as in Lemma 2.9. Set $\varphi_{K}(x)=\eta\left(|x|^{2} / K\right)$ and $\zeta_{M}(x)=\eta\left(\left(1+|x|^{2}\right)^{\kappa} / M\right)$ on $\mathbb{R}^{N}$. 
For each $K \in \mathbb{N}$ we find a number $M=M(K)$ such that $\zeta_{M}(x)=1$ if $|x|^{2}<2 K$.

Fix $t \in \bigcap_{K \in \mathbb{N}}\left(J_{\psi \varphi_{K}}^{\sigma^{1}} \bigcap J_{\psi \varphi_{K}}^{\sigma^{2}}\right)$, where the sets $J_{\psi}^{\mu}$ are defined in Remark 1.2. Let $f$ be a smooth bounded solution to the finite-dimensional Cauchy problem

$$
\left\{\begin{array}{l}
\partial_{t} f+\zeta_{M} \sum_{i, j=1}^{N} a^{i j} \partial_{x_{i}} \partial_{x_{j}} f+\zeta_{M} \sum_{i=1}^{N} b_{\varepsilon}^{i} \partial_{x_{i}} f=0 \quad \text { on } \mathbb{R}^{N} \times(0, t), \\
f(t, x)=\psi(x)
\end{array}\right.
$$

Set $\mu=\sigma^{1}-\sigma^{2}$. The measure $\mu$ solves the Cauchy problem (1.1) with zero initial condition. Recall that $\zeta_{M}(x)=1$ if $\varphi_{K}(x) \neq 0$. Therefore,

$$
\int_{\mathbb{R}^{\infty}} \psi \varphi_{K} d \mu_{t}=\int_{0}^{t} \int_{\mathbb{R}^{\infty}}\left[\varphi_{K}\left\langle B-b_{\varepsilon}, \nabla_{x} f\right\rangle+f L \varphi_{K}+2\left\langle A \nabla_{x} f, \nabla_{x} \varphi_{K}\right\rangle\right] d \mu_{s} d s .
$$

Applying Lemma 2.9 we have the estimate

$$
|f(x, s)| \leq 1, \quad\left|\nabla_{x} f(x, s)\right|^{2} \leq e^{\left(C_{\varepsilon}+1\right)\left(T_{0}-s\right)} V_{\varepsilon}(x) / 2
$$

Hence

$$
\begin{aligned}
\int_{\mathbb{R}^{\infty}} \psi d \mu_{t} \leq 2 \int_{0}^{t} \int_{\mathbb{R}^{\infty}} & {\left[\left|B-b_{\varepsilon}\right| V_{\varepsilon}^{1 / 2} e^{\left(C_{\varepsilon}+1\right)\left(T_{0}-s\right) / 2}+\left|L \varphi_{K}\right|\right.} \\
& \left.+2\left|A \nabla \varphi_{K}\right| e^{\left(C_{\varepsilon}+1\right)\left(T_{0}-s\right) / 2} V_{\varepsilon}^{1 / 2}\right] d \sigma_{s} d s
\end{aligned}
$$

Letting $K \rightarrow+\infty$ we find that

$$
\int_{\mathbb{R}^{\infty}} \psi d \mu_{t} \leq 2 \int_{0}^{t} \int_{\mathbb{R}^{\infty}}\left|B-b_{\varepsilon}\right| V^{1 / 2} e^{\left(C_{\varepsilon}+1\right)\left(T_{0}-s\right) / 2} d \sigma_{s} d s<2 \varepsilon .
$$

Since $\varepsilon>0$ was arbitrary, we obtain

$$
\int_{\mathbb{R}^{\infty}} \psi d \mu_{t} \leq 0
$$

Replacing $\psi$ by $-\psi$ we arrive at the equality

$$
\int_{\mathbb{R}^{\infty}} \psi d \mu_{t}=0
$$

Therefore,

$$
\int_{\mathbb{R}^{\infty}} \psi d \sigma_{t}^{1}=\int_{\mathbb{R}^{\infty}} \psi d \sigma_{t}^{2}
$$

for almost every $t$. Thus, $\sigma^{1}=\sigma^{2}$. 
Example 2.10 (Reaction diffusion equations). Let us return to the situation of Example 2.4, but now we assume that there exists a sequence of smooth bounded functions $f_{n}(z, u, t)$ such that $\lim _{n \rightarrow \infty} f_{n}(z, u, t)=f(z, u, t)$ for every $u, t, z$ and

$$
\left|f_{n}(z, u, t)\right| \leq C_{1}+C_{1}|u|^{m}, \quad\left(f_{n}(z, u, t)-f_{n}(z, v, t)\right)(u-v) \leq C_{2}|u-v|^{2},
$$

where $C_{1}$ and $C_{2}$ do not depend on $n$. Assume also that $a^{i j}=\left\langle S e_{i}, e_{j}\right\rangle_{2}$ for some symmetric nonnegative operator $S$ on $L^{2}[0,1]$, which can be degenerate (unlike the case of Example 2.4). Then there exists at most one probability solution $\mu$ of the Cauchy problem for the Fokker-Planck-Kolmogorov equation $\partial_{t} \mu=L^{*} \mu$ such that

$$
\int_{0}^{T_{0}} \int_{L^{2}[0,1]}\|u\|_{2 m}^{m} \mu_{t}(d u) d t<\infty .
$$

The same conclusion is true if $A=\left(a^{i j}\right)$ is a nonconstant matrix satisfying condition $\left(\mathrm{A}^{\prime}\right)$ and there exists a constant $C_{1}$ such that for every natural number $N$ and every $(x, t) \in \mathbb{R}^{N} \times\left[0, T_{0}\right]$ we have

$$
\sum_{i, j, k \leq N}\left|\partial_{x_{k}} \sigma_{N}^{i j}(x, t)\right|^{2} \leq C_{1}
$$

Proof. Set $F^{i}(u, t)=\left\langle f(\cdot, u(\cdot), t), e_{i}\right\rangle_{2}, F_{n}^{i}(u, t)=\left\langle f_{n}(\cdot, u(\cdot), t), e_{i}\right\rangle_{2}, F_{n}(u, t)=$ $\left(F_{n}^{i}(u, t)\right)_{i=1}^{\infty}$, and extend all these maps to all of $\mathbb{R}^{\infty} \times\left[0, T_{0}\right]$ by zero. According to our assumptions and the dominated convergence theorem we have

$$
\lim _{n \rightarrow \infty} \int_{0}^{T_{0}} \int_{L^{2}[0,1]}\left\|F(u, t)-F_{n}(u, t)\right\|_{l^{2}} \mu_{t}(d u) d t=0 .
$$

Let $P_{N} u:=u_{1} e_{1}+\ldots+u_{N} e_{N}$. The above equality shows that for each $\varepsilon>0$ and $d \geq 1$ there exist numbers $n$ and $N>d$ such that

$$
\int_{0}^{T_{0}} \int_{L^{2}[0,1]}\left\|F(u, t)-F_{n}\left(P_{N} u, t\right)\right\|_{l^{2}} \mu_{t}(d u) d t<\varepsilon .
$$

Note that the condition

$$
\left(f_{n}(z, u, t)-f_{n}(z, v, t)\right)(u-v) \leq C_{2}|u-v|^{2}
$$

implies that

$$
\sum_{i, j \leq N} \partial_{u_{i}} F_{n}^{j}\left(P_{N} u, t\right) h_{i} h_{j} \leq C_{2}|h|^{2}, \quad h=\left(h_{i}\right) \in \mathbb{R}^{N}
$$

Hence Theorem 2.5 with $V \equiv 1$ implies uniqueness.

Below for simplicity the integral of the product of an integrable function $f_{1}$ and a bounded function $f_{2}$ is denoted by $\left\langle f_{1}, f_{2}\right\rangle_{2}$. 
Example 2.11 (Stochastic Burgers equation). Suppose that $\left\{e_{k}\right\}$ is an eigenbasis of the Laplacian on $L^{2}[0,1]$ with zero boundary condition, i.e., $D^{2} e_{k}=-\lambda_{k} e_{k}$, $\lambda_{k}>0$. Set

$$
B(u)(z)=D^{2} u(z)+D\left(u^{2}(z)\right),
$$

that is,

$$
B^{i}(u)=-\lambda_{i} u_{i}-\left\langle u^{2}, D e_{i}\right\rangle_{2}, u \in L^{2}[0,1], u_{i}=\left\langle u, e_{i}\right\rangle_{2} .
$$

Assume that $a^{i j}=\left\langle S e_{i}, e_{j}\right\rangle_{2}$ for some symmetric nonnegative operator $S$ on $L^{2}[0,1]$ with finite trace $(\operatorname{tr} S<\infty)$. Set

$$
L \varphi=\sum_{i, j=1}^{\infty} a^{i j} \partial_{e_{i}} \partial_{e_{j}} \varphi+\sum_{i=1}^{\infty} B^{i} \partial_{e_{i}} \varphi
$$

where $\partial_{e_{i}}$ is the partial derivative along the vector $e_{i}$. Let $H_{0}^{1}$ be the space of all absolutely continuous functions $u$ on $[0,1]$ such that $u(0)=u(1)=0$ and $\|u\|_{H_{0}^{1}}:=\left\|u^{\prime}\right\|_{2}<\infty$. Then there exists at most one probability solution $\mu$ of the Cauchy problem for the Fokker-Planck-Kolmogorov equation $\partial_{t} \mu=L^{*} \mu$ such that

$$
\int_{0}^{T_{0}} \int_{L^{2}[0,1]}\|u\|_{H_{0}^{1}}^{2} e^{\delta\|u\|_{2}^{2}} \mu_{t}(d u) d t<\infty
$$

for some $\delta>0$ (which may depend on $\mu$ ).

Proof. We apply Example 2.8(ii). Recall that the matrix $\left(a^{i j}\right)$ has to satisfy the following condition for some $\varepsilon_{0}>0$ :

$$
\varepsilon_{0}\left(\left\langle A_{N} x, x\right\rangle+|x|^{2}\right) \leq\|x\|_{l_{\lambda}^{2}}^{2}, \quad x \in \mathbb{R}^{N} .
$$

This is equivalent to

$$
\varepsilon_{0}\left(\langle S u, u\rangle_{2}+\|u\|_{2}^{2}\right) \leq\|u\|_{H_{0}^{1}}^{2}, \quad u=x_{1} e_{1}+\cdots+x_{N} e_{N},
$$

which is true for sufficiently small $\varepsilon_{0}$. We fix $\varepsilon_{0} \in(0, \delta)$. Set

$$
F^{i}(u):=\left\langle u^{2}, D e_{i}\right\rangle_{2} \quad \text { for } u \in L^{2}
$$

and extend $F^{i}$ by zero to all other $u=\left(u_{k}\right)$ in $\mathbb{R}^{\infty}$. Let

$$
\begin{gathered}
F(u)=\left(F^{i}(u)\right)_{i=1}^{\infty}, \quad P_{N} u:=u_{1} e_{1}+\ldots+u_{N} e_{N}, \\
b^{k}\left(u_{1}, \ldots, u_{N}\right):=-\lambda_{k} u_{k}+F^{k}\left(P_{N} u\right), \quad k \leq N .
\end{gathered}
$$

Note that

$$
\|F(u)\|_{l^{2}}=\left\|\left(u^{2}\right)^{\prime}\right\|_{2}=2\left\|u u^{\prime}\right\|_{2} \leq 2\|u\|_{H_{0}^{1}}^{2} .
$$

Hence

$$
\lim _{N \rightarrow \infty} \int_{0}^{T_{0}} \int_{L^{2}[0,1]}\left\|F(u)-F\left(P_{N} u\right)\right\|_{l^{2}} e^{\delta\|u\|_{2}^{2}} \mu_{t}(d u) d t=0
$$


It is easy to see that $\left|b^{k}(u)\right| \leq C_{1}(N)+C_{2}(N)\left\|P_{N} u\right\|_{2}^{2}$ and $\left\langle F\left(P_{N} u\right), P_{N} u\right\rangle_{2} \leq 0$. Moreover, for every $\gamma \in(0,1)$ we have the inequalities

$$
\sum_{i, k \leq N} \partial_{u_{i}} F^{k}\left(P_{N} u\right) h_{i} h_{k} \leq\|h\|_{l_{\lambda}^{2}}+\left(\gamma\left\|P_{N} u\right\|_{H_{0}^{1}}^{2}+C_{\gamma}\right)|h|^{2}, \quad h=\left(h_{i}\right) \in \mathbb{R}^{N} .
$$

Set $\theta\left(P_{N} u\right)=\gamma\left\|P_{N} u\right\|_{H_{0}^{1}}^{2}+C_{\gamma}$ and $C_{0}=C_{\gamma}+\operatorname{tr} S$ (we recall that $\operatorname{tr} S<\infty$ ). In order to apply Example 2.8(ii) we choose $\gamma<2^{-1} \delta\left(\varepsilon_{0}-\delta\right)$.

\section{Example 2.12 (Mixed Burgers/reaction diffusion type equations).}

(i) In the situation of the previous example we consider the operator $L$ with the drift coefficient of the form

$$
B(u)(z)=D^{2} u(z)+D\left(u^{2}(z)\right)-u^{2 m+1}(z), \quad m \in \mathbb{N},
$$

that is,

$$
B^{i}(u)=-\lambda_{i} u_{i}-\left\langle u^{2}, D e_{i}\right\rangle_{2}-\left\langle u^{2 m+1}, e_{i}\right\rangle_{2} .
$$

Assume that $a^{i j}$ satisfies the assumptions in the previous example. Then there exists at most one probability solution $\mu$ of the Cauchy problem for the Fokker-Planck-Kolmogorov equation $\partial_{t} \mu=L^{*} \mu$ such that

$$
\int_{0}^{T_{0}} \int_{L^{2}[0,1]}\left[\|u\|_{4 m+2}^{2 m+1}+\|u\|_{H_{0}^{1}}^{2}\right] e^{\delta\|u\|_{2}^{2}} \mu_{t}(d u) d t<\infty
$$

for some $\delta>0$ (which may depend on $\mu$ ). Note that the measure $\mu_{t}$ is concentrated on $H_{0}^{1}$ for a.e. $t$.

(ii) In the situation of Example 2.11 we consider the operator $L$ with the drift coefficient of the form

$$
B(u)(z)=D^{2} u(z)+D\left(u^{m}(z)\right)-u^{2 l+1}(z), \quad 2 \leq m \leq l+1, \quad m, l \in \mathbb{N}
$$

that is,

$$
B^{i}(u)=-\lambda_{i} u_{i}-\left\langle u^{m}, D e_{i}\right\rangle_{2}-\left\langle u^{2 l+1}, e_{i}\right\rangle_{2} .
$$

Assume also that $a^{i j}=0$ if $i \neq j$ and that $\sum_{i=1}^{\infty} a^{i i}<\infty$. Then there exists at most one probability solution $\mu$ of the Cauchy problem for the Fokker-PlanckKolmogorov equation $\partial_{t} \mu=L^{*} \mu$ such that

$\int_{0}^{T_{0}} \int_{L^{2}((0,1))}\left[\|u\|_{4 l+2}^{2 l+1}+\|u\|_{H_{0}^{1}}+\left\|u^{m}\right\|_{H_{0}^{1}}\right] \exp \left(\kappa^{\prime}\left(\|u\|_{2 l+2}^{2 l+2}+\|u\|_{2}^{2}\right)\right) \mu_{t}(d u) d t<\infty$

for some $\kappa^{\prime}>0$ (which may depend on $\mu$ ). As above, the measure $\mu_{t}$ is concentrated on $H_{0}^{1}$ for a.e. $t$. This partially improves the results in [26] (where the last term in the equation is of linear growth). 
Proof. (i) We apply Example 2.8(ii). Note that as in the above example the matrix $\left(a^{i j}\right)$ satisfies all conditions in Example 2.8(ii). Let $\psi_{M} \in C^{\infty}\left(\mathbb{R}^{1}\right), \psi(s)=$ $-\psi(-s), 0 \leq \psi^{\prime} \leq 1, \psi_{M}(s)=s$ if $|s| \leq M-1$ and $\psi_{M}(s)=M$ if $s>M+1$. Set

$$
\begin{aligned}
F^{i}(u) & :=-\left\langle u^{2}, D e_{i}\right\rangle_{2}-\left\langle u^{2 m+1}, e_{i}\right\rangle_{2}, \quad F_{M}^{i}(u):=-\left\langle u^{2}, D e_{i}\right\rangle_{2}-\left\langle\psi_{M}(u)^{2 m+1}, e_{i}\right\rangle_{2}, \\
P_{N} u & :=u_{1} e_{1}+\cdots+u_{N} e_{N}, \quad b^{k}\left(u_{1}, \ldots, u_{N}\right):=-\lambda_{k} u_{k}+F_{M}^{k}\left(P_{N} u\right) .
\end{aligned}
$$

As above, we define all these functions by zero if $u$ is not in $L^{2}[0,1]$. Note that

$$
\|F(u)\|_{l^{2}} \leq 2\|u\|_{H_{0}^{1}}^{2}+\|u\|_{4 m+2}^{2 m+1}
$$

and the same is true for $F_{M}(u)$ in place of $F(u)$. Hence

$$
\lim _{N \rightarrow \infty}\left(\lim _{M \rightarrow \infty} \int_{0}^{T_{0}} \int_{L^{2}[0,1]}\left\|F(u)-F_{M}\left(P_{N} u\right)\right\|_{l^{2}} e^{\delta\|u\|_{2}^{2}} \mu_{t}(d u) d t\right)=0 .
$$

It is easy to see that $\left|b^{k}(u)\right| \leq C_{1}(N)+C_{2}(N)\left\|P_{N} u\right\|_{2}^{2}$. Recall that $\psi_{M}^{\prime} \geq 0$ and $\psi_{M}(s)=-\psi_{M}(-s)$. Hence $\left\langle F_{M}\left(P_{N} u\right), P_{N} u\right\rangle_{2} \leq 0$. For every $\gamma \in(0,1)$ we have

$$
\sum_{i, k \leq N} \partial_{u_{i}} F_{M}^{k}\left(P_{N} u\right) h_{i} h_{k} \leq\|h\|_{l_{\lambda}^{2}}+\left(\gamma\left\|P_{N} u\right\|_{H_{0}^{1}}^{2}+C_{\gamma}\right)|h|^{2}, \quad h=\left(h_{i}\right) \in \mathbb{R}^{N} .
$$

Set $\theta\left(P_{N} u\right)=\gamma\left\|P_{N} u\right\|_{H_{0}^{1}}^{2}+C_{\gamma}$ and $C_{0}=C_{\gamma}+\operatorname{tr} S$ (we recall that $\operatorname{tr} S<\infty$ ). In order to apply Example 2.8(ii) we choose $\gamma<2^{-1} \delta\left(\varepsilon_{0}-\delta\right)$.

(ii) We check condition $\left(\mathrm{B}^{\prime}\right)$. Let $\zeta \in C_{0}^{\infty}(\mathbb{R}), \zeta(x)=1$ if $|x| \leq 1, \zeta(x)=0$ if $|x| \geq 0,0 \leq \zeta \leq 1,\left|\zeta^{\prime}\right| \leq 1$. Set

$$
\zeta_{j}(x)=\zeta(x / j), \quad \psi_{j}(x)=\int_{0}^{x} \zeta_{j}(s) d s .
$$

We observe that $\psi_{j}(x)=x$ if $|x| \leq j,\left|\psi_{j}(x)\right| \leq|x|,\left|\psi_{j}^{\prime}\right| \leq 1,\left|\psi_{j}^{\prime \prime}(x)\right| \leq$ $I_{\{j<|x|<2 j\}}(x) / j$. Set

$$
\begin{gathered}
F_{j}^{k}(u)=-\left\langle\psi_{j}(u)^{m}, D e_{k}\right\rangle_{2}-\left\langle u^{2 l+1}, e_{k}\right\rangle_{2}, \quad P_{N} u=u_{1} e_{1}+\cdots+u_{N} e_{N}, \\
b^{k}\left(u_{1}, u_{2}, \ldots, u_{N}\right)=-\lambda_{k} u_{k}+F_{j}^{k}\left(P_{N} u\right) .
\end{gathered}
$$

Since

$$
\int_{0}^{1}\left|\left(m \psi_{j}(u)^{m-1} \psi_{j}^{\prime}(u)\right)^{\prime}\right|^{2} d x \leq C \int_{0}^{1}\left|\left(u^{m-1}\right)^{\prime}\right|^{2} d x
$$

with some $C$ independent of $j$, it is easy to see that for every $\gamma \in(0,1)$ there exists a number $C_{\gamma}>0$ (independent of $N$ ) such that

$$
\sum_{i, k \leq N} \partial_{u_{i}} F^{k}\left(u^{N}\right) h_{i} h_{k} \leq \gamma\|h\|_{l_{\lambda}^{2}}+\left(\gamma\left\|\left(P_{N} u\right)^{m-1}\right\|_{H_{0}^{1}}^{2}+C_{\gamma}\right)|h|^{2}, \quad h=\left(h_{i}\right) \in \mathbb{R}^{N} .
$$


We shall now construct a suitable Lyapunov function $V$ of the form $V=\exp (\alpha G)$, where

$$
G(u)=\frac{1}{2 l+2} \int_{0}^{1} u^{2 l+2} d x+\frac{M}{2} \int_{0}^{1} u^{2} d x
$$

and $\alpha$ and $M$ will be picked below. We have

$$
\begin{aligned}
\partial_{e_{i}} G(u) & =\int_{0}^{1} u^{2 l+1} e_{i} d x+M \int_{0}^{1} u e_{i} d x, \\
\partial_{e_{j}} \partial_{e_{i}} G(u) & =(2 l+1) \int_{0}^{1} u^{2 l} e_{i} e_{j} d x+M \delta_{i j} .
\end{aligned}
$$

Taking into account that $e_{i}^{\prime \prime}=-\lambda_{i}^{2} e_{i}$, we obtain

$$
\begin{aligned}
L G= & \sum_{i=1}^{n}\left(a^{i i} \partial_{e_{i}}^{2} G+b^{i} \partial_{e_{i}} G\right)=\sum_{i=1}^{n} a^{i i}(2 l+1) \int_{0}^{1} u^{2 l} e_{i}^{2} d x+M \sum_{i=1}^{n} a^{i i} \\
& +\sum_{i=1}^{n} \int_{0}^{1} u e_{i}^{\prime \prime} d x\left(\int_{0}^{1} u^{2 l+1} e_{i} d x+M \int_{0}^{1} u e_{i} d x\right) \\
& +m \sum_{i=1}^{n} \int_{0}^{1} \psi_{j}(u)^{m-1} \psi_{j}^{\prime}(u) u^{\prime} e_{i} d x\left(\int_{0}^{1} u^{2 l+1} e_{i} d x+M \int_{0}^{1} u e_{i} d x\right) \\
& -\sum_{i=1}^{n} \int_{0}^{1} u^{2 l+1} e_{i} d x\left(\int_{0}^{1} u^{2 l+1} e_{i} d x+M \int_{0}^{1} u e_{i} d x\right) .
\end{aligned}
$$

Therefore, since $\left|\psi_{j}(u)\right| \leq|u|$ and $\left|\psi_{j}^{\prime}\right| \leq 1$, we have

$$
\begin{aligned}
L G \leq & \sum_{i=1}^{\infty} a^{i i}\left(M+(2 l+1) \int_{0}^{1} u^{2 l} d x\right) \\
& -(2 l+1) \int_{0}^{1} u^{2 l}\left|u^{\prime}\right|^{2} d x-M \int_{0}^{1}\left|u^{\prime}\right|^{2} d x \\
& +\frac{m}{2 \alpha} \int_{0}^{1} u^{2 m-2}\left|u^{\prime}\right|^{2} d x+\frac{m \alpha}{2} \sum_{i=1}^{n}\left(\int_{0}^{1} u^{2 l+1} e_{i} d x\right)^{2} \\
& -M \int_{0}^{1} u^{2 l+2} d x-\sum_{i=1}^{n}\left(\int_{0}^{1} u^{2 l+1} e_{i} d x\right)^{2} .
\end{aligned}
$$

Note that $u^{2 m-2} \leq C(\delta)+\delta u^{2 l}$, since $2 m-2<2 l$. Taking $\delta$ small enough and $M$ large enough we obtain

$$
L G \leq C_{1}-C_{2} W,
$$

where $C_{1}, C_{2}>0$ are constants and

$$
W=\int_{0}^{1}\left|u^{\prime}\right|^{2} d x+\int_{0}^{1} u^{2 l}\left|u^{\prime}\right|^{2} d x+\int_{0}^{1} u^{2 l+2} d x+\sum_{i=1}^{n}\left(\int_{0}^{1} u^{2 l+1} e_{i} d x\right)^{2} .
$$


Hence

$$
L \exp (\alpha G) \leq \alpha L G+2 \alpha^{2} \sum_{i=1}^{\infty} a^{i i}\left(\sum_{i=1}^{n}\left(\int_{0}^{1} u^{2 l+1} e_{i} d x\right)^{2}+M\|u\|_{2}^{2}\right) .
$$

For $\alpha$ small enough we obtain

$$
L \exp (\alpha G) \leq\left(C_{3}-C_{4} W\right) \exp (\alpha G), \quad\left(\|u\|_{4 l+2}^{2 l+1}+\left\|u^{m}\right\|_{H_{0}^{1}}\right) \exp (\alpha G) \in L^{1}(\mu) .
$$

Note that $\left\|u^{m-1}\right\|_{H_{0}^{1}} \leq C\left(\left\|u^{l+1}\right\|_{H_{0}^{1}}+\|u\|_{H_{0}^{1}}\right)$, where $C$ depends on $l$ and $m$. Let us also observe that

$$
\begin{aligned}
|b|^{2} & =\sum_{i=1}^{n}\left|b^{i}\right|^{2} \leq 2 \sum_{i=1}^{n}\left(\left\langle u, e_{i}^{\prime \prime}\right\rangle^{2}+\left\langle\psi_{j}(u)^{m}, e_{i}^{\prime}\right\rangle^{2}+\left\langle u^{2 l+1}, e_{i}\right\rangle^{2}\right) \\
& \leq C(N)\left(\|u\|_{2}+\left\|u^{m}\right\|_{2}+\sum_{i=1}^{n}\left(\int_{0}^{1} u^{2 l+1} e_{i} d x\right)^{2}\right) .
\end{aligned}
$$

Taking $\delta_{N}$ sufficiently small we obtain $\delta_{N}\left|b\left(P_{N} u\right)\right|^{2}\left(1+\left\|P_{N} u\right\|_{2}^{2}\right)^{-1} \leq C_{4} W / 2$. Choosing $\gamma$ small enough we have

$$
L_{A_{N}, b} V\left(P_{N} u\right) \leq\left(C_{0}-\gamma\left\|\left(P_{N} u\right)^{m-1}\right\|_{H_{0}^{1}}^{2}-\delta_{N}\left|b\left(P_{N} u\right)\right|\left(1+\left\|P_{N} u\right\|_{2}^{2}\right)^{-1}\right) V\left(P_{N} u\right)
$$

for some number $C_{0}>0$. Note that $C_{0}$ does not depend on $N$ and we can omit the term $e^{C_{0}(T-t) / 2}$ in condition $\left(\mathrm{B}^{\prime}\right)(\mathrm{i})$.

Finally, we have to verify that for any given $\varepsilon>0$ and any $d \in \mathbb{N}$ there are $j$ and $N>d$ such that

$$
\int_{0}^{T_{0}} \int_{L^{2}[0,1]}\left\|F_{j}\left(P_{N} u\right)-F(u)\right\|_{l^{2}} \exp \left(\alpha G\left(P_{N} u\right)\right) \mu_{t}(d u) d t<\varepsilon .
$$

By a classical result (see [27, page 59]), for each $p>1$ there is a number $C(p)$ such that $\left\|P_{N} u\right\|_{p} \leq C(p)\|u\|_{p}$ and $\lim _{N \rightarrow \infty}\left\|P_{N} u-u\right\|_{p}=0$. Therefore, making $\alpha$ smaller, it is enough to get the above estimate for $G$ in place of $G \circ P_{N}$. Furthermore, we have

$$
\|F(u)\|_{l^{2}} \leq\left\|u^{m}\right\|_{H_{0}^{1}}+\|u\|_{4 l+2}^{2 l+1}, \quad\left\|F_{j}(u)\right\|_{l^{2}} \leq\left\|u^{m}\right\|_{H_{0}^{1}}+\|u\|_{4 l+2}^{2 l+1}
$$

and $\left\|F_{j}(u)-F(u)\right\|_{l^{2}} \rightarrow 0$ as $j \rightarrow \infty$ for any $u \in H_{0}^{1}$ such that $u^{m} \in H_{0}^{1}$, so by the Lebesgue dominated convergence theorem

$$
\lim _{j \rightarrow \infty} \int_{0}^{T_{0}} \int_{L^{2}[0,1]}\left\|F_{j}(u)-F(u)\right\|_{l^{2}} \exp (\alpha G) \mu_{t}(d u) d t=0 .
$$


For fixed $j$ we have

$$
\left\|F_{j}\left(P_{N} u\right)\right\|_{l^{2}} \leq C(j, m)\left\|P_{N} u\right\|_{H_{0}^{1}} \leq C(j, m)\|u\|_{H_{0}^{1}}, \quad\left\|F_{j}(u)\right\|_{l^{2}} \leq C(j, m)\|u\|_{H_{0}^{1}},
$$

hence

$$
\lim _{N \rightarrow \infty} \int_{0}^{T_{0}} \int_{L^{2}[0,1]}\left\|F_{j}\left(P_{N} u\right)-F_{j}(u)\right\|_{l^{2}} \exp (\alpha G) \mu_{t}(d u) d t=0 .
$$

Therefore, given $\varepsilon>0$, we can find $j$ such that

$$
\int_{0}^{T_{0}} \int_{L^{2}[0,1]}\left\|F_{j}(u)-F(u)\right\|_{l^{2}} \exp (\alpha G) \mu_{t}(d u) d t<\varepsilon / 2,
$$

next we take $N_{j}$ such that

$$
\int_{0}^{T_{0}} \int_{L^{2}[0,1]}\left\|F_{j}\left(P_{N} u\right)-F_{j}(u)\right\|_{l^{2}} \exp (\alpha G) \mu_{t}(d u)<\varepsilon / 2,
$$

so that

$$
\int_{0}^{T_{0}} \int_{L^{2}[0,1]}\left\|F_{j}\left(P_{N} u\right)-F(u)\right\|_{l^{2}} \exp (\alpha G) \mu_{t}(d u) d t<\varepsilon .
$$

Hence Theorem 2.5 implies uniqueness of a solution.

It should be noted that such "mixed" equations have been considered in [26] and [34]; the example above shows that the explained method covers both types of equations simultaneously.

Example 2.13 (Stochastic $2 d$-Navier-Stokes equation). Let us consider the space $V_{2}$ of $\mathbb{R}^{2}$-valued mappings $u=\left(u^{1}, u^{2}\right)$ such that $u^{j} \in H_{0}^{2,1}(D)$ and $\operatorname{div} u=0$, where $D \subset \mathbb{R}^{2}$ is a bounded domain with smooth boundary. The space $V_{2}$ is equipped with its natural Hilbert norm $\|u\|_{V_{2}}$ defined by

$$
\|u\|_{V_{2}}^{2}:=\sum_{j=1}^{2}\left\|\nabla_{z} u^{j}\right\|_{2}^{2}
$$

Let $H$ be the closure of $V_{2}$ in $L^{2}\left(D, \mathbb{R}^{2}\right)$ and let $P_{H}$ denote the orthogonal projector on $H$ in $L^{2}\left(D, \mathbb{R}^{2}\right)$. It is known (see [28]) that there exists an orthonormal basis $\left\{\eta_{n}\right\}$ in $H$ formed by eigenfunctions of $\Delta$ with eigenvalues $-\lambda_{n}<0$ such that $\eta_{n} \in V_{2}$. Recall that $\left\langle P_{H} w, \eta_{n}\right\rangle_{2}=\left\langle w, \eta_{n}\right\rangle_{2}$ for any $w \in L^{2}\left(D, \mathbb{R}^{d}\right)$. Set

$$
B^{n}(u, t)=\left\langle u, \Delta \eta_{n}\right\rangle_{2}-\sum_{j=1}^{2}\left\langle P_{H} u^{j} \partial_{z_{j}} u, \eta_{n}\right\rangle_{2}=\left\langle u, \Delta \eta_{n}\right\rangle_{2}-\sum_{j=1}^{2}\left\langle\partial_{z_{j}} u, u^{j} \eta_{n}\right\rangle_{2}
$$


whenever $u \in V_{2}$ and $B^{n}(u, t)=0$ otherwise. These functions are continuous on balls in $V_{2}$ with respect to the topology of $L^{2}\left(D, \mathbb{R}^{2}\right)$, which easily follows from the compactness of the Sobolev embedding $H^{2,1}(D) \rightarrow L^{2}(D)$. Consider the operator

$$
L \varphi(u, t)=\sum_{i, j}^{\infty} a^{i j} \partial_{\eta_{i}} \partial_{\eta_{j}} \varphi(u, t)+\sum_{n=1}^{\infty} B^{n}(u, t) \partial_{\eta_{n}} \varphi(u, t)
$$

Assume that $a^{i j}=\left\langle S \eta_{i}, \eta_{j}\right\rangle_{2}$ for some symmetric nonnegative bounded operator $S$ on $H$. Suppose also that $\sum_{i} a^{i i} \lambda_{i}<\infty$. Then there exists at most one probability solution $\mu$ of the Cauchy problem for the Fokker-Planck-Kolmogorov equation $\partial_{t} \mu=L^{*} \mu$ such that for some $\delta>0$

$$
\int_{0}^{T_{0}} \int_{H}\left(1+\|\Delta u\|_{2}^{2}\right) e^{\delta\|u\|_{V_{2}}^{2}} \mu_{t}(d u) d t<\infty
$$

where we set $\|\Delta u\|_{2}=\infty$ if $u^{i} \notin H^{2,2}(D)$.

Proof. We apply Example 2.8(iii). Recall that the matrix $\left(a^{i j}\right)$ has to satisfy the following condition for some $\varepsilon_{0}>0$ :

$$
\varepsilon_{0} \sum_{i, j \leq N} a^{i j} \lambda_{i} \lambda_{j} x_{i} x_{j}+\varepsilon_{0}|x|^{2} \leq \sum_{i \leq N} \lambda_{i}^{2} x_{i}^{2}
$$

that is equivalent to the estimate

$$
\varepsilon_{0}\left(\langle S \Delta u, \Delta u\rangle_{2}+\|u\|_{2}^{2}\right) \leq\|\Delta u\|_{2}^{2},
$$

which is true for sufficiently small $\varepsilon_{0}$. Set

$$
F^{n}(u)=-\sum_{j=1}^{2}\left\langle\partial_{z_{j}} u, u^{j} \eta_{n}\right\rangle_{2}, \quad u \in V_{2}
$$

Note that $\left|F^{n}(u)\right| \leq C_{1}(n)+C_{2}(n)\|u\|_{2}^{2}$, since $F^{n}(u)=\sum_{j=1,2}\left\langle u, u_{j} \partial_{z_{j}} \eta_{n}\right\rangle_{2}$ due to the condition that $\operatorname{div} u=0$. It is well-known that there exists a constant $C_{1}>0$ such that for every function $g \in H_{0}^{2,1}(D) \cap H^{2,2}(D)$ we have

$$
\|g\|_{2,2} \leq C_{1}\|\Delta g\|_{2} \text {. }
$$

Moreover, for every $g \in H^{2,2}(D)$ and some constant $C_{2}>0$ (since we deal with $d=2$ ) we have

$$
\|g\|_{4} \leq C_{2}\|g\|_{2,1}
$$

Hence

$$
\begin{aligned}
\|F(u)\|_{l^{2}}^{2} & \leq \int_{D}\left|\nabla_{z} u(z)\right|^{2}|u(z)|^{2} d z \leq\left(\int_{D}\left|\nabla_{z} u(z)\right|^{4} d z\right)^{1 / 2}\left(\int_{D}|u(z)|^{4} d z\right)^{1 / 2} \\
& \leq C_{1}^{2} C_{2}^{4}\left(1+\|\Delta u\|_{2}^{2}\right)\|u\|_{V_{2}}^{2} .
\end{aligned}
$$


Let $P_{N} u=u_{1} \eta_{1}+\cdots+u_{N} \eta_{N}$. We have

$$
\lim _{N \rightarrow \infty} \int_{0}^{T_{0}} \int_{H}\left\|F(u)-F\left(P_{N} u\right)\right\|_{l^{2}} e^{\delta\|u\|_{V_{2}}^{2} / 2} \mu_{t}(d u) d t=0 .
$$

It is known (see, e.g., [15, Proposition 6.3]) that in the considered case $d=2$ we have the inequality

$$
\left\langle F\left(P_{N} u\right), \Delta P_{N} u\right\rangle_{2}=0
$$

which gives the condition $\sum_{i=1}^{N} \lambda_{i} f^{i}(x, t) x_{i}=0$ required in Example 2.8(iii) (now $F^{i}$ plays the role of $\left.f^{i}\right)$. In addition, for every $\gamma \in(0,1)$ we have

$$
\sum_{i, j \leq N} \partial_{u_{i}} F^{j}\left(P_{N} u\right) h_{i} h_{j} \leq\left(C_{\gamma}+\gamma\left\|\Delta P_{N} u\right\|_{l^{2}}^{2}\right)|h|^{2}+\|h\|_{l_{\lambda}^{2}}^{2}, \quad h=\left(h_{i}\right) .
$$

Set $\theta\left(P_{N} u\right)=C_{\gamma}+\gamma\left\|\Delta P_{N} u\right\|_{l^{2}}^{2}$ and $C_{0}=C_{\gamma}+\sum_{i=1}^{\infty} a^{i i} \lambda_{i}$ (we recall that $\left.\sum_{i=1}^{\infty} a^{i i} \lambda_{i}<\infty\right)$. In order to apply Example 2.8(iii) we choose $\gamma<2^{-1} \delta\left(\varepsilon_{0}-\delta\right)$. In Example 3.5 we consider a more general equation.

It is worth noting that the last example applies to degenerate coefficients $A$, in particular, to $A$ identically zero, which gives uniqueness for the so-called continuity equation corresponding to $2 d$-Navier-Stokes equation.

In the next section we show that the considered classes of uniqueness are not empty.

\section{Existence of solutions}

First we would like to mention that if the stochastic equation associated to our Fokker-Planck-Kolmogorov equation has a solution in the sense of Stroock-Varadhan's martingale problem, then one immediately gets a solution to the FPK-equation. But uniqueness of solutions for a martingale problem does not imply uniqueness for the corresponding FPK-equation.

In this section we purely analytically prove the following existence result generalizing a result from [4] (where only a sketch of the proof of a weaker result was given, namely, $V$ was the square of the norm).

Let $\left\{e_{n}\right\}$ be an orthonormal basis in $l^{2}$. The linear span of $e_{1}, \ldots, e_{n}$ is denoted by $H_{n}$.

Let $T_{0}>0$ and let $a^{i j}: \mathbb{R}^{\infty} \times\left[0, T_{0}\right] \rightarrow \mathbb{R}^{1}$ and $B^{i}: \mathbb{R}^{\infty} \times\left(0, T_{0}\right) \rightarrow \mathbb{R}^{1}$ be Borel functions. Suppose that the matrices $\left(a^{i j}\right)_{i, j \leq n}$ are symmetric nonnegative for all $n$. Set

$$
L \varphi(x, t):=\sum_{i, j=1}^{n} a^{i j}(x, t) \partial_{e_{i}} \partial_{e_{j}} \varphi(x, t)+\sum_{i=1}^{n} B^{i}(x, t) \partial_{e_{i}} \varphi(x, t),(x, t) \in \mathbb{R}^{\infty} \times\left(0, T_{0}\right)
$$

for functions $\varphi$ that are smooth functions of the variables $x_{1}, \ldots, x_{n}, t$.

Let $B_{n}:=\left(B^{1}, \ldots, B^{n}\right)$ and $P_{n} x=\left(x_{1}, \ldots, x_{n}\right)$. 
A Borel function $\Theta: \mathbb{R}^{\infty} \rightarrow[0,+\infty]$ such that the sublevel sets $\{\Theta \leq R\}$, where $R<\infty$, are compact is called a compact function. For example, one can take any numbers $\alpha_{i}>0$ and set $\Theta(x)=\sum_{i=1}^{\infty} \alpha_{i}^{2} x_{i}^{2}$.

Theorem 3.1. Suppose that there exists a compact function $\Theta: \mathbb{R}^{\infty} \rightarrow[0,+\infty]$, finite on each $H_{n}$ and such that the functions $a^{i j}$ and $B^{i}$ are continuous in $x$ on all the sets $\{\Theta \leq R\}$, and there exist numbers $M_{0}, C_{0} \geq 0$ and a Borel function $V: \mathbb{R}^{\infty} \rightarrow[1,+\infty]$ whose sublevel sets $\{V \leq R\}$ are compact and whose restrictions to $H_{n}$ are of class $C^{2}$ and such that for all $x \in H_{n}, n \geq 1, t \in\left(0, T_{0}\right)$ one has

$$
\sum_{i, j=1}^{n} a^{i j}(x, t) \partial_{e_{i}} V(x) \partial_{e_{j}} V(x) \leq M_{0} V(x)^{2}, \quad L V(x, t) \leq C_{0} V(x)-\Theta(x)
$$

Assume also that there exist constants $C_{i} \geq 0$ and $k_{i} \geq 0$ such that for all $i$ and $j \leq i$ one has

$$
\left|a^{i j}(x, t)\right|+\left|B^{i}(x, t)\right| \leq C_{i} V(x)^{k_{i}}(1+\delta(\Theta(x)) \Theta(x)),(x, t) \in \mathbb{R}^{\infty} \times\left(0, T_{0}\right),
$$

where $\delta$ is a bounded nonnegative Borel function on $[0,+\infty)$ with $\lim _{s \rightarrow \infty} \delta(s)=$ 0 . Then, for every Borel probability measure $v$ on $\mathbb{R}^{\infty}$ such that

$$
W_{k}:=\sup _{n}\left\|V^{k} \circ P_{n}\right\|_{L^{1}(v)}<\infty \quad \forall k \in \mathbb{N},
$$

the Cauchy problem (1.1) with initial distribution $v$ has a solution of the form $\mu=$ $\mu_{t} d t$ with Borel probability measures $\mu_{t}$ on $\mathbb{R}^{\infty}$ such that

$$
\int_{\mathbb{R}^{\infty}} V^{k} d \mu_{t}+k \int_{0}^{t} \int_{\mathbb{R}^{\infty}} V^{k-1} \Theta d \mu_{s} d s \leq N_{k} W_{k} \quad \forall k \in \mathbb{N}, t \in\left(0, T_{0}\right),
$$

where $N_{k}:=M_{k} e^{M_{k}}+1, \quad M_{k}=k\left(C_{0}+(k-1) M_{0}\right)$. In particular, $\mu_{t}(V<$ $\infty)=1$ for all $t$ and $\mu_{t}(\Theta<\infty)=1$ for almost all $t$.

Proof. For every fixed $n$ let $a_{n}^{i j}$ denote the restriction of $a^{i j}$ to $H_{n} \times\left(0, T_{0}\right)$ and set $A_{n}:=\left(a_{n}^{i j}\right)_{i, j \leq n}$. Denote by $v_{n}$ the projection of $v$ on $H_{n}$. We show that there exist Borel probability measures $\mu_{t, n}$ on $H_{n}$ such that the measure $\mu_{n}:=\mu_{t, n} d t$ solves the Cauchy problem with coefficients $A_{n}$ and $B_{n}$ on $H_{n} \times\left(0, T_{0}\right)$ and initial distribution $v_{n}$. To this end we consider the Lyapunov function $V_{m}(x)=V(x)^{m}$ on $H_{n}$, where $m \geq 1$. Letting $M_{m}:=m\left(C_{0}+(m-1) M_{0}\right)$, we obtain

$$
\begin{aligned}
L V_{m} & =m V^{m-1}\left(L V+(m-1) V^{-1} \sum_{i, j=1}^{n} a^{i j} \partial_{e_{i}} V \partial_{e_{j}} V\right) \\
& \leq m V^{m-1}\left(C_{0} V-\Theta+(m-1) M_{0} V\right) \leq M_{m} V^{m}-m V^{m-1} \Theta .
\end{aligned}
$$


Since the function $V_{m}$ is $v_{n}$-integrable, we can apply the existence result from [3] and obtain the desired probability measures $\mu_{t, n}$ on $H_{n}$ (with $\mu_{0, n}=v_{n}$ ) such that the function

$$
t \mapsto \int_{H_{n}} \zeta(x) \mu_{t, n}(d x)
$$

is continuous on $\left[0, T_{0}\right)$ for every $\zeta \in C_{0}^{\infty}\left(H_{n}\right)$. Moreover, by [4, Lemma 1] (see also [3, Lemma 2.2]), for each $m \geq 1$ and

$$
N_{m}:=M_{m} e^{M_{m}}+1, \quad M_{m}=m\left(C_{0}+(m-1) M_{0}\right)
$$

the following estimate holds for almost all $t \in\left(0, T_{0}\right)$ :

$$
\begin{aligned}
& \int_{H_{n}} V_{m}(x) \mu_{t, n}(d x)+m \int_{0}^{t} \int_{H_{n}} V_{m-1}(x) \Theta(x) \mu_{s, n}(d x) d s \\
& \leq N_{m} \int_{H_{n}} V_{m}(x) v_{n}(d x) \leq N_{m}+N_{m} W_{m} .
\end{aligned}
$$

Therefore, by Fatou's theorem and the above stated continuity of $t \mapsto \mu_{t, n}$ it follows that (3.4) holds for all $t \in\left[0, T_{0}\right)$. Indeed, we replace $V_{m}$ and $\Theta V_{m-1}$ in the left-hand side by $\min \left(k, V_{m}\right)$ and $\min \left(k, \Theta V_{m-1}\right)$, obtain the desired estimate for all $t \in\left[0, T_{0}\right)$ keeping $k$ fixed and then let $k \rightarrow \infty$.

Suppose now that $\zeta \in C_{0}^{\infty}\left(\mathbb{R}^{d}\right)$. Let us identify $H_{n}$ with $\mathbb{R}^{n}$. If $n \geq d$, then $\zeta$ regarded as a function on $\mathbb{R}^{n}$ belongs to the class $C_{b}^{\infty}\left(\mathbb{R}^{n}\right)$. Let $m=$ $\max \left(k_{1}, \ldots, k_{d}\right)$. Then we have the estimate

$$
|L \zeta(x, t)| \leq K+K V_{m}(x)+K V_{m}(x) \delta(\Theta(x)) \Theta(x),(x, t) \in \mathbb{R}^{n} \times\left(0, T_{0}\right),
$$

where $K$ is some number which depends on $\zeta$ (but is independent of $n$ since $\zeta$ is a function of $x_{1}, \ldots, x_{d}$ ). Therefore, by approximation, inequality (3.4) and Lebesgue's dominated convergence theorem we have

$$
\int_{H_{n}} \zeta(x) \mu_{t, n}(d x)=\int_{0}^{t} \int_{H_{n}} L \zeta(x, s) \mu_{s, n}(d x) d s+\int_{H_{n}} \zeta(x) v_{n}(d x),
$$

because, according to [3], this identity holds for all $\zeta \in C_{0}^{\infty}\left(\mathbb{R}^{n}\right)$, hence in our situation it remains valid also for all $\zeta \in C_{b}^{\infty}\left(\mathbb{R}^{n}\right)$. Letting

$$
\varphi_{n}(t):=\int_{H_{n}} \zeta(x) \mu_{t, n}(d x), t \in\left[0, T_{0}\right),
$$

we see from (3.4), (3.6) that the function $\varphi_{n}$ is Lipschitzian (one can also show that it is everywhere differentiable in $\left.\left(0, T_{0}\right)\right)$ and $(3.5)$ yields that

$$
\left|\varphi_{n}^{\prime}(t)\right| \leq \int_{H_{n}}|L \zeta(x, t)| \mu_{t, n}(d x) \leq K_{\zeta} \int_{H_{n}}\left[1+V_{m-1}(x) \Theta(x)\right] \mu_{t, n}(d x)
$$


with some number $K_{\zeta}$ that does not depend on $n$ (but only on $\zeta$ ). Therefore, by (3.4) the functions $\varphi_{n}$ possess uniformly bounded variations, hence there is a subsequence in $\left\{\varphi_{n}\right\}$ convergent pointwise on $\left[0, T_{0}\right)$. We may assume that this is true for the whole sequence. Moreover, we can do this in a such a way that this pointwise convergence holds for every function $\zeta$ from a fixed countable family $\mathcal{F}$ with the following property: the weak convergence of a uniformly tight sequence of probability measures on $\mathbb{R}^{\infty}$ follows from convergence of their integrals of every function in $\mathcal{F}$.

It follows from (3.4) and the compactness of the sets $\left\{V_{m} \leq R\right\}$ and $\{\Theta \leq R\}$ that, for every fixed $t \in\left(0, T_{0}\right)$, the sequence of measures $\mu_{t, n}$ is uniformly tight on $\mathbb{R}^{\infty}$ (see [2, Example 8.6.5]). Hence we can find a subsequence, denoted for simplicity by the same indices $n$, such that $\left\{\mu_{t, n}\right\}$ converges weakly on $\mathbb{R}^{\infty}$ for every rational $t \in\left(0, T_{0}\right)$. However, since we have ensured convergence of $\varphi_{n}(t)$ at every $t \in\left[0, T_{0}\right)$ for every $\zeta \in \mathcal{F}$, we see that $\left\{\mu_{t, n}\right\}$ converges weakly for every $t \in\left[0, T_{0}\right)$.

Estimate (3.3) follows from (3.4) taking into account that $V \geq 1$ and $\Theta \geq 0$ are lower semicontinuous, hence $V^{k}$ and $V^{k-1} \Theta$ are lower continuous as well.

The family of measures $\mu_{t}$ obtained in this way is the desired solution. Indeed, let us fix $\zeta \in C_{0}^{\infty}\left(\mathbb{R}^{d}\right)$. We have to show that the integrals of $L \zeta(x, t)$ over $\mathbb{R}^{\infty} \times$ $(0, T), T<T_{0}$, with respect to $\mu_{n}$ converge to the integral with respect to $\mu=$ $\mu_{t} d t$. This amounts to establishing such convergence for all functions $f=\partial_{x_{i}} \zeta B^{i}$ and $f=a^{i j} \partial_{x_{j}} \partial_{x_{i}} \zeta$. Suppose we are able to show this for the functions $f_{N}=$ $\max (\min (f, N),-N)$. Then (3.2) and (3.4) enable us to extend the same to the original function $f$, because for every $\varepsilon>0$ these estimates give a number $N$ such that the integral of $|f| I_{|f|>N}$ with respect to $\mu_{t, n} d t$ is less than $\varepsilon$. Indeed, it suffices to show that the integral of $G:=V^{k}(1+\delta(\Theta) \Theta)$ over the set $\{G \geq N\}$ with respect to $\mu_{t, n} d t$ does not exceed $\varepsilon$ for $N$ sufficiently large. Take $n_{1}$ such that $1 / n_{1}+\delta(s)<c \varepsilon$ for all $s \geq n_{1}$, where $c>0$ is so small that $c N_{k+1} W_{k+1}<1 / 2$. We may assume that $\delta \leq 1$. We have

$$
\begin{aligned}
\int_{0}^{T_{0}} \int_{\left\{\Theta \geq n_{1}\right\}} G d \mu_{t, n} d t & =\int_{0}^{T_{0}} \int_{\left\{\Theta \geq n_{1}\right\}}\left(\Theta^{-1}+\delta(\Theta)\right) V^{k} \Theta d \mu_{t, n} d t \\
& \leq c \varepsilon \int_{0}^{T_{0}} \int_{H_{n}} V^{k} \Theta d \mu_{t, n} d t \leq \varepsilon / 2
\end{aligned}
$$

For any $N \geq n_{1}$ and $t<T_{0}$ we have

$$
\int_{\left\{G \geq N, \Theta \leq n_{1}\right\}} G d \mu_{t, n} \leq\left(1+n_{1}\right) \int_{\left\{V^{k} \geq N /\left(1+n_{1}\right)\right\}} V^{k} d \mu_{t, n} \leq N^{-1}\left(1+n_{1}\right)^{2} N_{k} W_{k},
$$

which can be made smaller than $\varepsilon / 2$ uniformly in $t<T_{0}$ for all $N$ sufficiently large.

Thus, it remains to justify the desired convergence in the case of $f_{N}$, which will be now denoted by $f$. We recall that the restriction of such a function $f$ to every set $\{\Theta \leq R\} \times\left(0, T_{0}\right)$ is continuous in the first variable. Dividing by $N$ we assume 
that $|f| \leq 1$. If $f$ were continuous in $x$ on the whole space, this would follow at once from the weak convergence of $\mu_{t, n}$ for every fixed $t$. Our situation reduces to this one in the standard way: given $\varepsilon>0$, we find $R$ so large that the set $\{\Theta \geq$ $R\} \times\left(0, T_{0}\right)$ has measure less than $\varepsilon$ with respect to all measures $\mu_{t, n} d t$ and $\mu_{t} d t$. By our assumption the set $\Omega=\{\Theta \leq R\}$ is compact in $\mathbb{R}^{\infty}$. The mapping $t \mapsto$ $f(\cdot, t)$ from $\left(0, T_{0}\right)$ to $C(\Omega)$ is Borel measurable. By Dugundji's theorem (see [14, Chapter III, Section 7]), there is a linear extension operator $E: C(\Omega) \rightarrow C_{b}\left(\mathbb{R}^{\infty}\right)$ such that $E \varphi(x)=\varphi(x)$ for all $\varphi \in C(\Omega), x \in \Omega$ and $\|E \varphi\|_{\infty}=\|\varphi\|_{\infty}$. Letting $g(x, t)=E f(\cdot, t)(x)$, we obtain a Borel function (since it is Borel measurable in $t$ and continuous in $x$, see [2, Lemma 6.4.6]) such that $|g| \leq 1$ and $g(t, x)=f(t, x)$ for all $x \in \Omega$. The integral of $g$ with respect to $\mu_{t, n} d t$ converges to the integral of $g$ with respect to $\mu_{t} d t$ and the integrals of $|f-g|$ with respect to these measures do not exceed $\varepsilon$. Therefore, the measure $\mu=\mu_{t} d t$ satisfies our parabolic equation with initial distribution $v$.

The condition that $V \geq 1$ is taken just for simplicity of estimates: it can be replaced by $V \geq 0$ if we add constants in the right sides of (3.1) and (3.2).

In typical examples $V$ and $\Theta$ are quadratic functions (with added constants). For example, we shall use $V(x)=\sum_{i=1}^{\infty} \beta_{i} x_{i}^{2}+1$ and $\Theta(x)=\sum_{i=1}^{\infty} \alpha_{i} x_{i}^{2}$. There is also a version of this theorem applicable to exponents of quadratic functions (the first inequality in (3.1) is not suitable for such functions).

Theorem 3.2. Suppose that in Theorem 3.1 condition (3.1) is replaced by

$$
L V(x, t) \leq V(x)-V(x) \Theta(x)
$$

and (3.2) is replaced by

$$
\begin{aligned}
& \left|a^{i j}(x, t)\right|+\left|B^{i}(x, t)\right| \leq C_{i}(1+\delta(V(x) \Theta(x)) V(x) \Theta(x)), \\
& (x, t) \in \mathbb{R}^{\infty} \times\left(0, T_{0}\right) .
\end{aligned}
$$

Then, for every Borel probability measure $v$ on $\mathbb{R}^{\infty}$ with

$$
W_{1}:=\sup _{n}\left\|V \circ P_{n}\right\|_{L^{1}(v)}<\infty
$$

the Cauchy problem (1.1) with initial distribution $v$ has a solution of the form $\mu=$ $\mu_{t} d t$ with Borel probability measures $\mu_{t}$ on $\mathbb{R}^{\infty}$ such that for $t \in\left(0, T_{0}\right)$

$$
\int_{\mathbb{R}^{\infty}} V d \mu_{t}+\int_{0}^{t} \int_{\mathbb{R}^{\infty}} V \Theta d \mu_{s} d s \leq 4 W_{1} .
$$

Proof. The reasoning is much the same as in the previous theorem, but we use only one Lyapunov function $V$ and use (3.7) in place of (3.4) to obtain the estimate

$$
\begin{aligned}
& \int_{H_{n}} V(x) \mu_{t, n}(d x)+\int_{0}^{t} \int_{H_{n}} V(x) \Theta(x) \mu_{s, n}(d x) d s \\
& \leq(e+1) \int_{H_{n}} V(x) \mu_{0, n}(d x) \leq 4 W_{1} .
\end{aligned}
$$


Another place where some difference arises is the estimate of the integral of $f I_{|f|>N}$, where $|f|$ is estimated by $C(1+\delta(V \Theta) V \Theta)$, but this is easily done by using the previous inequality and the condition that $\delta(s) \rightarrow 0$ as $s \rightarrow \infty$.

Let us apply the last theorem to the Fokker-Planck-Kolmogorov equation associated with the stochastic Burgers type equations (see Example 2.11).

Example 3.3 (Stochastic Burgers equation). Let us return to the situation of Example 2.11. Let $u$ belong to the linear span of $\left\{e_{k}\right\}$. Note that

$$
\langle B(u), u\rangle_{2}=-\|u\|_{H_{0}^{1}}^{2} \cdot
$$

Let $V(u)=\exp \left(\delta\|u\|_{2}^{2}\right)$. We have

$$
L V(u) \leq 2 \delta\left(\operatorname{tr} S+2 \delta\langle S u, u\rangle_{2}-\|u\|_{H_{0}^{1}}^{2}\right) V(u)
$$

Taking $\delta<\varepsilon_{0} / 4$ we obtain

$$
L V(u) \leq(1-\Theta(u)) V(u), \quad \Theta(u)=1-2 \delta \operatorname{tr} S+\delta\|u\|_{H_{0}^{1}}^{2} .
$$

In addition, $\left|B^{k}(u)\right| \leq C(k)+C(k)\|u\|_{2}^{2}$. According to Theorem 3.2 for every initial condition $v$ with $\exp \left(\delta\|u\|_{2}^{2}\right) \in L^{1}(v)$ there exists a probability solution $\mu$ of the Cauchy problem $\partial_{t} \mu=L^{*} \mu,\left.\mu\right|_{t=0}=v$ such that

$$
\int_{0}^{T_{0}} \int_{L^{2}[0,1]}\|u\|_{H_{0}^{1}}^{2} \exp \left(\delta\|u\|_{2}^{2}\right) \mu_{t}(d u) d t<\infty .
$$

According to Example 2.11 this $\mu$ is the unique probability solution with this property.

Example 3.4. Let us return to the situation of Example 2.12, where we deal only with the more general case (ii). Assume that $a^{i j}=0$ if $i \neq j$ and that $\sum_{i} a^{i i}<\infty$. Let $u$ belong to the linear span of $\left\{e_{k}\right\}$. Using the same Lyapunov function $V=$ $\exp (\varepsilon G)$ as in that example, we obtain $L V \leq\left(C_{3}-C_{4} W_{n}\right) V$, where

$$
W_{n}(u)=\int_{0}^{1}\left|u^{\prime}\right|^{2} d x+\int_{0}^{1} u^{2 l}\left|u^{\prime}\right|^{2} d x+\int_{0}^{1} u^{2 l+2} d x+\sum_{i=1}^{n}\left(\int_{0}^{1} u^{2 l+1} e_{i} d x\right)^{2}
$$

for all $u \in H_{m}$ with $m \geq n$. According to Theorem 3.2 for every initial condition $v$ with

$$
V \in L^{1}(v)
$$

there exists a probability solution $\mu$ of the Cauchy problem $\partial_{t} \mu=L^{*} \mu,\left.\mu\right|_{t=0}=v$ such that

$$
\int_{0}^{T_{0}} \int_{L^{2}[0,1]} W_{n}(u) V(u) \mu_{t}(d u) d t \leq C,
$$


where $C$ is independent of $n$. By Fatou's theorem

$$
\int_{0}^{T_{0}} \int_{L^{2}[0,1]} V(u)\left[\left\|u^{\prime}\right\|_{2}^{2}+\left\|u^{l} u^{\prime}\right\|_{2}^{2}+\left\|u^{l+1}\right\|_{2}^{2}+\left\|u^{2 l+1}\right\|_{2}^{2}\right] \mu_{t}(d u) d t \leq C .
$$

We want to apply Example 2.12(ii) to show that this $\mu$ is the unique probability solution with this property. To this end it suffices to note that $\left\|u^{m}\right\|_{H_{0}^{1}} \leq C\|u\|_{H_{0}^{1}}^{2}+$ $C\left\|u^{l} u^{\prime}\right\|_{2}^{2}$, since $2 \leq m<l+1$. This partially generalizes a result in [32] (where restrictions on $m$ and $l$ are imposed, see [32, Condition (F1)] for details).

Let us apply the existence theorems to the Fokker-Planck-Kolmogorov equation associated with the stochastic Navier-Stokes equation in any dimension (a special case has been considered in Example 2.13).

Example 3.5. The stochastic equation of Navier-Stokes type is considered in the space $V_{2}$ of $\mathbb{R}^{d}$-valued mappings $u=\left(u^{1}, \ldots, u^{d}\right)$ such that $u^{j} \in H_{0}^{2,1}(D)$ and $\operatorname{div} u=0$, where $D \subset \mathbb{R}^{d}$ is a bounded domain with smooth boundary. The space $V_{2}$ is equipped with its natural Hilbert norm $\|u\|_{V_{2}}$ defined by

$$
\|u\|_{V_{2}}^{2}:=\sum_{j=1}^{d}\left\|\nabla_{z} u^{j}\right\|_{2}^{2}
$$

Let $H$ be the closure of $V_{2}$ in $L^{2}\left(D, \mathbb{R}^{d}\right)$ and let $P_{H}$ denote the orthogonal projection on $H$ in $L^{2}\left(D, \mathbb{R}^{d}\right)$. The stochastic Navier-Stokes equation is formally written as

$d u(z, t)=\sqrt{2} d W(z, t)+P_{H}\left[\Delta_{z} u(z, t)-\sum_{j=1}^{d} u^{j}(z, t) \partial_{z_{j}} u(z, t)+F(z, u(z, t), t)\right] d t$

where $W$ is a Wiener process of the form $W(z, t)=\sum_{n=1}^{\infty} \sqrt{\alpha_{n}} w_{n}(t) \eta_{n}(z)$, where

$$
\alpha_{n} \geq 0, \quad \sum_{n=1}^{\infty} \alpha_{n}<\infty
$$

$w_{n}$ are independent Wiener processes, and $\left\{\eta_{n}\right\}$ is an orthonormal basis in $H$, and

$$
F: D \times \mathbb{R}^{d} \times\left(0, T_{0}\right) \rightarrow \mathbb{R}^{d}
$$

is a bounded continuous mapping. No interpretation of this equation is needed for the sequel, it should be regarded only as a heuristic expression leading to a specific form of the corresponding elliptic operator. The case $F=0$ is the classical stochastic Navier-Stokes equation. Note that the action of $P_{H}$ in the right-hand side is defined in the natural way: $P_{H} \Delta_{z} u(z, t):=P_{H} \Delta_{z} u(\cdot, t)(z)$ and similarly for the other terms. Since the Laplacian $\Delta$ is not defined on all of $V_{2}$, this equation 
requires some interpretation. Our approach suggests the following procedure. It is known (see [28]) that there exists an orthonormal basis $\left\{\eta_{n}\right\}$ in $H$ formed by eigenfunctions of $\Delta$ with eigenvalues $-\lambda_{n}<0$ such that $\eta_{n} \in V_{2}$. Employing the fact that $\left\langle P_{H} w, \eta_{n}\right\rangle_{2}=\left\langle w, \eta_{n}\right\rangle_{2}$ for any $w \in L^{2}\left(D, \mathbb{R}^{d}\right)$, we introduce the "coordinate" functions

$$
\begin{aligned}
B^{n}(u, t) & =\left\langle u, \Delta \eta_{n}\right\rangle_{2}-\sum_{j=1}^{d}\left\langle P_{H}\left(u^{j} \partial_{z_{j}} u\right), \eta_{n}\right\rangle_{2}+\left\langle P_{H} F(\cdot, u(\cdot, t), t), \eta_{n}\right\rangle_{2} \\
& =\left\langle u, \Delta \eta_{n}\right\rangle_{2}-\sum_{j=1}^{d}\left\langle\partial_{z_{j}} u, u^{j} \eta_{n}\right\rangle_{2}+\left\langle F(\cdot, u(\cdot, t), t), \eta_{n}\right\rangle_{2} .
\end{aligned}
$$

These functions are defined by the last line on all of $V_{2}$. They are continuous on balls in $V_{2}$ with respect to the topology of $L^{2}\left(D, \mathbb{R}^{d}\right)$, which follows by the compactness of the embedding of $H^{2,1}(D) \rightarrow L^{2}(D)$. Choosing a Wiener process of the above form, we arrive at the operator

$$
L \varphi(u, t)=\sum_{n=1}^{\infty} \alpha_{n} \partial_{\eta_{n}}^{2} \varphi(u, t)+\sum_{n=1}^{\infty} B^{n}(u, t) \partial_{\eta_{n}} \varphi(u, t)
$$

Since for every $u$ from the linear span of $\left\{\eta_{n}\right\}$ one has

$$
\sum_{n=1}^{\infty} \sum_{j=1}^{d}\left\langle u, \eta_{n}\right\rangle_{2}\left\langle\partial_{z_{j}} u, u^{j} \eta_{n}\right\rangle_{2}=\sum_{j=1}^{d}\left\langle u, u^{j} \partial_{z_{j}} u\right\rangle_{2}=-\frac{1}{2} \int_{D}|u(z)|^{2} \operatorname{div} u(z) d z=0
$$

and $\langle\Delta u, u\rangle_{2}=-\|u\|_{V_{2}}^{2}$, we have the estimate

$$
\sum_{n=1}^{N}\left\langle u, \eta_{n}\right\rangle_{2} B^{n}(u, t) \leq C_{1}-C_{1}\|u\|_{V_{2}}^{2}
$$

for all $u$ in the linear span of $\eta_{1}, \ldots, \eta_{N}$, where $C_{1}$ is a constant independent of $N$. Clearly, we have also

$$
\left|B^{n}(u, t)\right| \leq C_{2}(n)+C_{2}(n)\|u\|_{2}^{2} .
$$

Therefore, by Theorem 3.1 applied with $\Theta(u)=C_{1}\|u\|_{V_{2}}^{2}$ and $V(u)=\|u\|_{2}^{2}+1$ (the above estimates along with convergence of the series of $\alpha_{n}$ mean that we have (3.1)) there is a probability measure $\mu=\mu_{t} d t$ on $V_{2} \times\left[0, T_{0}\right)$, such that $\mu_{t}(H)=1$ for all $t$ and $\mu_{t}\left(V_{2}\right)=1$ for almost all $t$, and solving the Cauchy problem (1.1) with any initial distribution $v$ for which $\|u\|_{2}^{k} \in L^{1}(v)$ for all $k$. This settles the easier problem of existence.

It should be also noted that Flandoli and Gatarek [21] proved (under the stated assumptions) the existence of a solution to the martingale problem associated with 
the operator $L$ such that this solution possesses all moments in $H$. One can show that the measure generated by this solution satisfies the Fokker-Planck-Kolmogorov equation in our sense.

Let us consider the $2 \mathrm{~d}-$ Navier-Stokes equation, i.e., $d=2$ and $F=0$, where we are able to establish also uniqueness. Recall that for every $u$ from the linear span of $\left\{\eta_{n}\right\}$ one has

$$
\sum_{n=1}^{\infty} \sum_{j=1}^{2}\left\langle u, \Delta \eta_{n}\right\rangle_{2}\left\langle\partial_{z_{j}} u, u^{j} \eta_{n}\right\rangle_{2}=0
$$

Set $V(u)=\exp \left(\delta\|u\|_{V_{2}}^{2}\right)$. Let $u$ be from the linear span of $\left\{\eta_{n}\right\}$. We have

$$
L V(u)=2 \delta\left(\sum_{n} \alpha_{n} \lambda_{n}+2 \delta \sum_{n} \alpha_{n} \lambda_{n}^{2} u_{n}^{2}-\sum_{n} \lambda_{n}^{2} u_{n}^{2}\right) V(u) .
$$

Assume that $\sum_{n=1}^{\infty} \alpha_{n} \lambda_{n}<\infty$. Hence for sufficiently small $\delta>0$

$$
L V(u) \leq(1-\Theta(u)) V(u), \quad \Theta(u)=1-\delta \sum_{n=1}^{\infty} \alpha_{n} \lambda_{n}+\delta\|\Delta u\|_{2}^{2},
$$

where $\Theta(u)=+\infty$ if $u^{i} \notin H^{2,2}(D)$. According to Theorem 3.2 for every initial condition $v$ with $\exp \left(\delta\|u\|_{V_{2}}^{2}\right) \in L^{1}(v)$ there exists a probability solution $\mu$ of the Cauchy problem $\partial_{t} \mu=L^{*} \mu,\left.\mu\right|_{t=0}=v$ such that

$$
\int_{0}^{T_{0}} \int_{H}\left(1+\|\Delta u\|_{2}^{2}\right) e^{\delta\|u\|_{V_{2}}^{2}} \mu_{t}(d u) d t<\infty .
$$

According to Example 2.13 this measure $\mu$ is the unique probability solution with this property.

Finally, we formulate one more existence and uniqueness result which is a combination of Theorem 3.1 and Theorem 2.3.

Corollary 3.6. Let $a^{i j}=0$ if $i \neq j$ and $a^{i i}=\alpha_{i}>0$. Suppose that the hypotheses of Theorem 3.1 are fulfilled with certain functions $V$ and $\Theta$. If there exists a Borel mapping $F=\left(F_{n}\right): \mathbb{R}^{\infty} \times\left(0, T_{0}\right) \rightarrow \mathbb{R}^{\infty}$ and numbers $p>0, C>0$ such that

$$
\|F(x, t)\|_{l_{\alpha}^{2}}^{2} \leq C V(x)^{p} \Theta(x)
$$

and for each natural number $n$ the difference $B^{n}(x, t)-F^{n}(x, t)$ depends only on $t$ and $x_{1}, x_{2}, \ldots, x_{n}$, then, for every initial condition $v$ with $V \in L^{k}(v)$ for every $k \geq 1$, the class $\mathcal{P}_{\nu}$ (see Theorem 2.3) consists of exactly one element.

Example 3.7. Let $a^{i j}=0$ if $i \neq j$ and $a^{i i}=\alpha_{i}>0$. Suppose that

$$
B^{n}(x, t)=-\beta_{n} x_{n}+F^{n}(x, t), \quad \text { where } \beta_{n}>0 .
$$


Let $\gamma_{n} \in(0,+\infty)$ be such that

$$
\sum_{n=1}^{\infty} \alpha_{n} \gamma_{n}<\infty
$$

Let

$$
V(x)=1+\sum_{n=1}^{\infty} \gamma_{n} x_{n}^{2}, \quad \Theta(x)=\sum_{n=1}^{\infty} \beta_{n} \gamma_{n} x_{n}^{2} .
$$

Let $c_{00}$ denote the subspace of all vectors $x \in \mathbb{R}^{\infty}$ with at most finitely many nonzero coordinates.

Suppose that a Borel mapping $F(\cdot, \cdot): \mathbb{R}^{\infty} \times\left(0, T_{0}\right) \rightarrow \mathbb{R}^{\infty}$ satisfies the following conditions: for each $t$ it is continuous in $x$ on every set $\{\Theta \leq R\}$ and there are numbers $\varepsilon \in(0,1), C_{1}>0, C_{2}>0$, and $p>0$ such that for all $t \in\left(0, T_{0}\right)$ and $x \in c_{00}$ one has

$\sum_{n=1}^{\infty} \gamma_{n} F^{n}(t, x) x_{n} \leq \varepsilon \Theta(x)+C_{1} V(x), \sum_{n=1}^{\infty} \alpha_{n}^{-1}\left|F^{n}(t, x)\right|^{2} \leq C_{2}(1+\Theta(x)) V(x)^{p}$.

Then, for every initial condition $v$ with $V \in L^{k}(v)$ for every $k \geq 1$, the class $\mathcal{P}_{v}$ consists of exactly one element.

Remark 3.8. As already noted, if the infinite-dimensional stochastic differential equation (SDE) associated to our Fokker-Planck-Kolmogorov equation has a solution in the sense of Stroock-Varadhan, then one gets a solution to the FPK-equation (but not vice versa). In contrast to that, uniqueness of solutions to the martingale problem does not imply the uniqueness of solutions to the FPK-equation, here the converse is true. Therefore, the existence parts in our Examples 3.3-3.5 can partly also be derived by probabilistic methods. It should also be pointed out that in these examples we always assume that $\left(a^{i j}\right)$ is trace class. For existence results by probabilistic means in case of Example 3.3 and the first part of Example 3.4 without this condition we refer to [24] and its recent improvement [34]. Furthermore, we believe that by a similar method as in [16] one can also prove uniqueness for the FPK-equation in the Burgers case (see Example 3.3) without the trace class condition. Finally, we point out that here we consider the Burgers case only on the bounded domain $D=(0,1) \subset \mathbb{R}$. If $D=\mathbb{R}$, existence, however, also holds. This follows from the probabilistic results in [25].

\section{References}

[1] S. AlbeVERIO and R. HøEGH-KROHn, Dirichlet forms and diffusion processes on rigged Hilbert spaces, Z. Wahrscheinlichkeitstheorie Verw. Gebiete 40 (1977), 1-57.

[2] V. I. BoGACHEV, "Measure Theory", Vol. 1, 2, Springer, Berlin-New York, 2007.

[3] V. I. Bogachev, G. DA Prato and M. RöcKNER, On parabolic equations for measures, Comm. Partial. Differential Equations 33 (2008), 397-418.

[4] V. I. Bogachev, G. Da Prato and M. Röckner, Parabolic equations for measures on infinite-dimensional spaces, Dokl. Russian Acad. Sci. 421 (2008), 439-444 (Russian); Dokl. Math. 78 (2008), 544-549. 
[5] V. Bogachev, G. Da Prato and M. RÖCKner, Fokker-Planck equations and maximal dissipativity for Kolmogorov operators with time dependent singular drifts in Hilbert spaces, J. Funct. Anal. 256 (2009), 1269-1298.

[6] V. Bogachev, G. DA Prato, M. Röckner and S. V. Shaposhnikov, On the uniqueness of solutions to continuity equations, J. Differential Equations (2015), doi:10.1016/ j.jde.2015.05.003

[7] V. BogacheV, G. DA Prato and M. RÖCKNER, Existence and uniqueness of solutions for Fokker-Planck equations on Hilbert spaces, J. Evol. Equ. 10 (2010), 487-509.

[8] V. Bogachev, G. DA Prato and M. RÖCKner, Uniqueness for solutions of FokkerPlanck equations on infinite dimensional spaces, Comm. Partial Diffential Equations 36 (2011), 925-939.

[9] V. I. BogacheV, G. Da Prato, M. Röckner and W. Stannat, Uniqueness of solutions to weak parabolic equations for measures, Bull. London Math. Soc. 39 (2007), 631-640.

[10] V. I. BOGACHEV, N. V. KRYLOV and M. RÖCKNER, On regularity of transition probabilities and invariant measures of singular diffusions under minimal conditions, Comm. Partial Differential Equations 26 (2001), 2037-2080.

[11] V. I. BOGACHEV, N. V. KRYLOV and M. RÖCKNER, Elliptic and parabolic equations for measures, Uspehi Mat. Nauk 64 (2009), 5-116 (Russian); Russian Math. Surveys 64 (2009), 973-1078.

[12] V. I. BogacheV, M. RÖCKNER and S. V. Shaposhnikov, Positive densities of transition probabilities of diffusion processes, Teor. Veroyatn. Primen. 53 (2008), 213-239 (Russian), Theory Probab. Appl. 53 (2009), 194-215.

[13] V. I. BogacheV, M. RÖCKNER and S. V. Shaposhnikov, On uniqueness problems related to the Fokker-Planck-Kolmogorov equation for measures, J. Math. Sci. (New York) 179 (2011), 759-773.

[14] K. BORSUK, "Theory of Retracts", Polish Scientific Publ., Warszawa, 1967.

[15] G. DA PRATO, "Kolmogorov Equations for Stochastic PDEs", Birkhäuser, Basel, 2004.

[16] G. DA PRATO and A. DEBUSSCHE, $m$-dissipativity of Kolmogorov operators corresponding to Burgers equations with space-time white noise, Ann. Probab. 41 (2013), 3306-3344.

[17] G. Da Prato, F. Flandoli, E. Priola and M. Röckner, Strong uniqueness for stochastic evolution equations in Hilbert spaces perturbed by a bounded measurable drift, Ann. Probab. 41 (2013), 3306-3344.

[18] S. D. Eidel'MAN, "Parabolic Systems", Nauka, Moscow, 1964 (Russian), Scripta Technica, London; North-Holland, Amsterdam-London; Wolters-Noordhoff, Groningen, 1969.

[19] A. FIgALLI, Existence and uniqueness of martingale solutions for SDEs with rough or degenerate coefficients, J. Funct. Anal. 254 (2008), 109-153.

[20] F. FLANDOLI, Random perturbations of PDEs and fluid dynamics models, In: "Lectures from the 40th Probability Summers School hold in Saint-Flour" 2010, Lecture Notes in Mathematics, Vol. 2015, Springer, Heidelberg, 2011, pp. X+176.

[21] F. FLANDOLI and D. GATAREK, Martingale and stationary solutions for stochastic NavierStokes equations, Probab. Theory Related Fields 102 (1995), 367-391.

[22] F. Flandoli and M. Romito, Markov selections for the 3D stochastic Navier-Stokes equations, Probab. Theory Related Fields 140 (2008), 407-458.

[23] A. Friedman, "Partial Differential Equations of Parabolic Type", Krieger, Malabar, Florida, 1983.

[24] I. GYÖNGY, Existence and uniqueness results for semilinear stochastic partial differential equations, Stoch. Processes Appl. 73 (1997), 271-299.

[25] I. GYÖNGY and D. NUALART, On the stochastic Burgers' equation in the real line, Ann. Probab. 27 (1999), 782-802.

[26] I. GYÖNGY and C. ROviRA, On $L^{p}$-solutions of semilinear stochastic equations, Stoch. Processes Appl. 90 (2000), 83-108.

[27] Y. KATZNELSON, "An Introduction to Harmonic Analysis", 3nd ed., Cambridge University Press, Cambridge, 2004. 
[28] O. A. Ladyzhenskaya, "The Mathematical Theory of Viscous Incompressible Flow", 2nd ed., Gordon and Breach, New York-Melbourne, 1969.

[29] C. LE BRIS and P.-L. LiONS, Existence and uniqueness of solutions to Fokker-Planck type equations with irregular coefficients, Comm. Partial Differential Equation 33 (2008), $1272-1317$.

[30] O. A. OlEINIK, On the smoothness of solutions of degenerating elliptic and parabolic equations, Dokl. Akad. Nauk SSSR 163 (1965), 577-580 (Russian).

[31] F. O. PORPER and S. D. EIDEL'MAN, Two-sided estimates of fundamental solutions of second-order parabolic equations and some application, Russian Math. Surveys 39 (1984), $119-178$.

[32] M. RÖCKNER and Z. SOBOL, Kolmogorov equations in infinite dimensions: well-posedness and regularity of solutions, with applications to stochastic generalized Burgers equations, Ann. Probab. 34 (2006), 663-727.

[33] M. RÖCKNER and T. ZHANG, Uniqueness of generalized Schrödinger operators and applications, J. Math. Anal. Appl. 415 (2014), 83-109.

[34] M. RÖCKNER, R. ZHU and X. ZHU, A note on stochastic semilinear equations and their associated Fokker-Planck equations, J. Math. Anal. Appl. 415 (2014), 83-109.

[35] S. V. SHAPOSHNIKOV, On the uniqueness of integrable and probability solutions to the Cauchy problem for the Fokker-Planck-Kolmogorov equations, Dokl. Ross. Akad. Nauk 439 (2011), 323-328 (Russian); Dokl. Math. 84 (2011), 565-570.

[36] D. W. Stroock and S. R. S. VARAdHan, "Multidimensional Diffusion Processes", Springer-Verlag, Berlin-New York, 1979.

Department of Mechanics and Mathematics Moscow State University 119991 Moscow, Russia

and

National Research University

Higher School of Economics

Moscow, Russia

vibogach@mail.ru

starticle@mail.ru

Scuola Normale Superiore di Pisa

Piazza dei Cavalieri 7

56126 Pisa, Italy

giuseppe.daprato@sns.it

Fakultät für Mathematik

Universität Bielefeld

D-33501 Bielefeld, Germany

roeckner@math.uni-bielefeld.de 